\title{
Stability at nonconforming grid interfaces for a high order discretization of the Schrödinger equation
}

October 7, 2011

\author{
A. Nissen* ${ }^{*}$ G. Kreiss ${ }^{\dagger}$ and M. Gerritsen ${ }^{\ddagger}$ \\ * Department of Information Technology, Uppsala University, Uppsala, \\ Sweden, anna.nissen@it.uu.se. \\ †Department of Information Technology, Uppsala University, Uppsala, \\ Sweden, gunilla.kreiss@it.uu.se. \\ $\ddagger$ Department of Energy Resources Engineering, Stanford University, \\ Stanford, CA, margot.gerritsen@stanford.edu.
}

\begin{abstract}
In this paper we extend the results from our earlier work on stable boundary closures for the Schrödinger equation using the summation-by-parts-simultaneous approximation term (SBP-SAT) method [8] to include stability and accuracy at non-conforming grid interfaces. Stability at the grid interface is shown by the energy method, and the estimates are generalized to multiple dimensions. The accuracy of the grid interface coupling is investigated using normal mode analysis for operators of 2 nd and 4 th order formal interior accuracy. We show that full accuracy is retained for the 2nd and 4th order operators. The accuracy results are extended to 6th and 8th order operators by numerical simulations, in which case two orders of accuracy is gained with respect to the lower order approximation close to the interface.
\end{abstract}




\section{Introduction}

Fundamental chemical reactions are governed by the Time-Dependent Schrödinger Equation (TDSE), which in one dimension is given by

$$
i \hbar \frac{\partial U}{\partial t}=-\frac{\hbar^{2}}{2 m} \frac{\partial^{2} U}{\partial x^{2}}+V U, \quad x \in \mathbb{R}, \quad t \geq 0
$$

where $\hbar$ is the reduced Planck's constant, $m$ is the mass of the system, and $i=\sqrt{-1}$. Equation (1.1) can be interpreted as describing the interaction between nuclei in a two-particle molecule, with $x$ the internuclear distance. The electron influence is modeled through the spatially dependent potential $V$. The potential can also be temporally dependent if interactions with exterior fields, such as laser pulses, are included in the model. The solution to equation (1.1), $U(x, t)$, is a complex valued function. However, $|U(x, t)|^{2}$ is real and of interest: it is the probablity density. The probability that the internuclear distance is in the interval $\left[x_{1}, x_{2}\right]$ at time $t$ is given by $\int_{-x_{1}}^{x_{2}}|U(x, t)|^{2} d x$. Physically reasonable solutions satisfy $\int_{-\infty}^{\infty}|U(x, t)|^{2} d x=1$ for any $t$. For this equation, it is easy to show that this integral is conserved.

The time-evolution of the internal motion in larger molecules can be described by a similar TDSE in higher dimensions, where each dimension corresponds to an internal degree of freedom. For example, the twodimensional TDSE

$$
i \hbar \frac{\partial U}{\partial t}=-\frac{\hbar^{2}}{2 m_{x}} \frac{\partial^{2} U}{\partial x^{2}}-\frac{\hbar^{2}}{2 m_{y}} \frac{\partial^{2} U}{\partial y^{2}}+V U, \quad x, y \in \mathbb{R}^{2}, \quad t \geq 0,
$$

can be used to model a molecule with an inner structure composed of two bond lengths, or one bond length and one bond angle. Numerically solving the TDSE, particularly in higher dimensions, is computationally very intense, and hence efficient numerical tools are of the essence.

One way to use computational resources more efficiently is through adaptive mesh refinement (AMR). In AMR, the computational grid is adapted dynamically. In areas where the solution changes rapidly, the computational grid is refined, and areas that no longer need a higher grid resolution are coarsened. The total number of grid points in a static grid increases exponentially with $N^{d}$, where $d$ is the dimensionality of the problem and $N$ the number of grid points in each dimension. Therefore, AMR is particularly attractive in higher dimensions and can lead to significant savings in computational work and memory requirements. Berger and Oliger developed a structured approach of adaptive mesh refinement (SAMR), based on the idea of multiple component grids for finite difference meth-

ods [1]. To allow for simpler data structures and reduce overhead time for 
grid-fitting, block-structured versions of SAMR have been developed [2]. In the block-structured approach, refinement is carried out concurrently for all points in a, typically rectangular, grid patch if the numerical solution fails to meet a user-defined local error tolerance. In order for such an approach to be successful, the artificial internal boundaries between adjoining grid blocks with different grid densities need to be treated carefully so as to preserve stability and accuracy properties [3]. In this paper we consider the numerical properties across nonconforming grid blocks (for which collocation points across grid boundaries do not match) for a finite difference discretization of the Schrödinger equation. We consider adjoining blocks where the refinement levels along the interface have a $2: 1$ ratio.

Spectral methods can be described through expansions based of smooth functions with global support. We expect the solutions to the TDSE to be smooth, and due to this the TDSE can often be accurately and efficiently discretized in space using spectral methods [4]. However, due to the global nature of the basis functions, the value of the derivative at a point in space often depends on the solution values at all other points in space, which in its turn make spectral methods difficult to combine with local grid refinement.

In contrast, high order finite difference methods can easily be combined with an AMR approach. The summation-by-parts-simultaneous approximation term (SBP-SAT) methodology is based on finite difference methods, with special boundary closures for which stability of the numerical approximations often can be shown $[5,6]$. Also, important properties of the single-block configuration, such as stability and accuracy, are usually preserved upon extension to multi-block geometries [7]. Until recently, however, it was not known how to couple neighboring nonconforming grid blocks in a stable way using the SBP-SAT technique, which impeded the use of local grid refinement techniques. However, this impediment was overcome by Mattsson and Carpenter, who constructed interpolation operators that together with commonly used SBP operators preserves stability between nonconforming grid blocks [7].

In an earlier work, we presented a framework for analyzing accuracy of stable boundary closures, and applied it to the Schrödinger equation [8]. In this paper we extend the results from [8] in the following ways. We demonstrate how to apply the SAT technique to yield stability at grid interfaces in one dimension. The stability results are then extended to nonconforming grid blocks in two dimensions using the interpolation operators constructed in [7]. We also consider the accuracy of stable grid couplings by the approach established in [8]. The methodology is based on a separation of interior truncation error and interface truncation error. The interior error is estimated by the energy method, whereas the interface error is investigated using normal mode analysis.

The outline of the paper is as follows. Estimates showing stability at 
interfaces between nonconforming grid blocks for an arbitrary number of dimensions are derived in section 2. The accuracy of the discretizations are investigated in section 3. Numerical experiments are presented in section 4 , and section 5 concludes with a discussion and an outline of future work.

\section{Stability of a grid interface}

We consider the Cauchy problem for the normalized Schrödinger equation $(\hbar=1,2 m=1)$ in one and more spatial dimensions. The potential $V$ does not affect the stability analysis, and for simplicity we set $V \equiv 0$. A forcing term $F$ is added to the right hand side of (1.1). This gives, in one dimension,

$$
U_{t}=i U_{x x}+F, x \in R, t \geq 0, U(x, 0)=f(x),\|U\|<\infty .
$$

In $N>1$ dimensions, we consider the normalized equation

$$
\begin{array}{r}
U_{t}=i U_{x x}+i \Delta_{y} U+F,(x, y) \in R^{N}, t \geq 0 \\
U(x, y, 0)=f(x, y),\|U\|<\infty .
\end{array}
$$

Here, $y$ is a $N-1$ vector, $\Delta_{y}$ is the corresponding Laplacian, and $\|\cdot\|$ denotes the standard $L_{2}$ norm.

\section{$2.1 \quad$ One dimension}

In the discrete setting we will use grid functions on the left grid $x_{-j}=$ $-j h_{L}, j=0,1,2, \ldots$, and on the right grid $x_{i}=i h_{R}, i=0,1,2, \ldots$, respectively. We denote by $\|\cdot\|_{h}$ the standard $l_{2}$ norm of these grid functions. We also define an inner product and norm for discrete complex valued grid functions $u, v \in \mathbb{C}^{N}$ as

$$
(u, v)_{P}=u^{*} P v, \quad\|u\|_{P}^{2}=u^{*} P u,
$$

where ${ }^{*}$ denotes the Hermitian transpose. In the case of real valued quantities ${ }^{*}$ is reduced to the transpose ${ }^{T} . P$ is a positive definite operator. Here, we will only consider diagonal P's. Note that with $P=h I$ we get the standard discrete norm.

Consider the two-half plane semi-discretization corresponding to the 
Cauchy problem (2.1),

$$
\begin{array}{r}
u_{t}=i Q_{L} u-\gamma_{L} P_{L}^{-1} S_{L}^{T} e_{0, L}\left(u_{0}-v_{0}\right)-\tau_{L} P_{L}^{-1} e_{0, L}\left(\left(S_{L} u\right)_{0}-\left(S_{R} v\right)_{0}\right)+\tilde{F}_{L}, \\
(2.4) \\
v_{t}=i Q_{R} v-\gamma_{R} P_{R}^{-1} S_{R}^{T} e_{0, R}\left(v_{0}-u_{0}\right)-\tau_{R} P_{R}^{-1} e_{0, R}\left(\left(S_{R} v\right)_{0}-\left(S_{L} u\right)_{0}\right)+\tilde{F}_{R}, \\
(2.5) \\
t \geq 0, u(0)=f_{1}, v(0)=f_{2},\|u(t)\|_{h}<\infty,\|v(t)\|_{h}<\infty,
\end{array}
$$

where $u, f_{1}, \tilde{F}_{L}$ and $v, f_{2}, \tilde{F}_{R}$ are grid functions on the left grid and on the right grid, respectively. A block interface is situated at $x=0$, as illustrated in figure 1. $Q_{L}$ and $Q_{R}$ are standard SBP operators approximating the second derivative, with interior order $2 m$ and interior stencil width $2 m+1$, see [9] for details. At $p$ points near the interface the second derivative approximation is of order $m$. The standard operators have $m=1,2,3,4$, and $p=1,4,6,8$, respectively, and correspond to diagonal norms. $P_{L}$ and $P_{R}$ are the diagonal operators corresponding to the norms on the left and right half-planes, respectively.

Note that the ordering of the equations in (2.4) and (2.5) is such that the last component of (2.4) and the first component of (2.5) both correspond to index 0 . Thus, we make a distinction between $e_{0, L}=(\ldots, 0,1)^{T}$ and $e_{0, R}=$ $(1,0, \ldots)^{T}$. Also, note that both $u_{0}=e_{0, L}^{T} u$ and $v_{0}=e_{0, R}^{T} v$ approximate $U(0, t)$. In the limit of $h_{L}, h_{R} \rightarrow 0, u_{0}=v_{0}$. The last row of $S_{L}$ and the first row of $S_{R}$, respectively, approximate the first derivative, such that both $\left(S_{L} u\right)_{0}=e_{0, L}^{T} S_{L} u$ and $\left(S_{R} v\right)_{0}=e_{0, R}^{T} S_{R} v$ approximate $U_{x}(0, t)$. The parameters $\gamma_{L}, \gamma_{R}, \tau_{L}, \tau_{R} \in \mathbb{C}$ are penalty terms that should be determined to yield a stable discretization (2.4), (2.5). In the stability analysis, there are no restrictions on the ratio $\frac{h_{L}}{h_{R}}$. However, in the accuracy analysis we will restrict the analysis to $\frac{h_{L}}{h_{R}}=2$. Other ratios are possible, but need to be considered separately.

The structures of $Q_{L}$ and $Q_{R}$ are given by

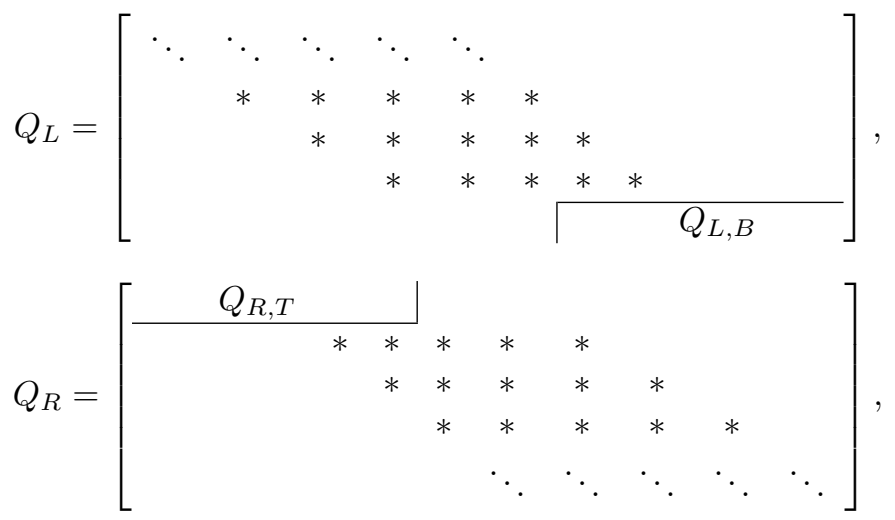


where $Q_{L, B}$ denotes the small matrix of size $p \times q$ in the lower right corner of $Q_{L}$ that contains the one-sided finite difference stencil used at the $p$ points closest to the grid interface. $Q_{R, T}$ is the corresponding matrix in the upper left corner of $Q_{R}$. For interior accuracy orders 2, 4,6, and 8, we have $q=3,6,9$ and 12. The asterisks in $Q_{L}$ and $Q_{R}$ correspond to the interior central stencil. Since $Q_{L}$ and $Q_{R}$ are SBP operators, they can be expressed as $Q_{L}=P_{L}^{-1}\left(-A_{L}+e_{0, L} e_{0, L}^{T} S_{L}\right), Q_{R}=P_{R}^{-1}\left(-A_{R}-e_{0, R} e_{0, R}^{T} S_{R}\right)$, where $A_{L / R}=A_{L / R}^{T}$. We will need this property in order to show stability for the grid interface coupling.

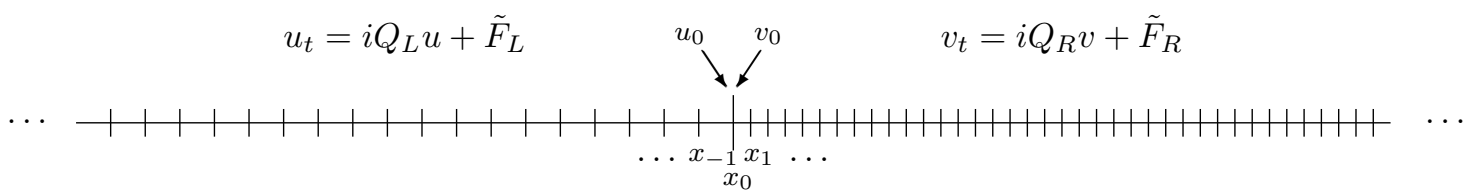

Figure 1: Grid interface coupling in one dimension.

Below follows a theorem with our first main result and the proof thereof.

Theorem 2.1. The SBT-SAT interface coupling (2.4), (2.5) is stable by the estimate

$\|u(t)\|_{P_{L}}^{2}+\|v(t)\|_{P_{R}}^{2} \leq\left\|f_{1}\right\|_{P_{L}}^{2}+\left\|f_{2}\right\|_{P_{R}}^{2}+e^{t_{0}} \int_{0}^{t_{0}}\left\|\tilde{F}_{L}(\tau)\right\|_{P_{L}}^{2}+\left\|\tilde{F}_{R}(\tau)\right\|_{P_{R}}^{2} d \tau$,

in any time interval $t \in\left[0, t_{0}\right]$, if the penalty parameters are chosen as

$$
\gamma_{L}=-\frac{i}{2}, \quad \gamma_{R}=\frac{i}{2}, \quad \tau_{L}=\frac{i}{2}, \quad \tau_{R}=-\frac{i}{2} .
$$

Proof: Multiplying equation (2.4) with $u^{*} P_{L}$, equation (2.5) with $v^{*} P_{R}$ and taking the real part gives 


$$
\begin{aligned}
& \frac{d}{d t}\|u\|_{P_{L}}^{2}+\frac{d}{d t}\|v\|_{P_{R}}^{2}=u^{*} P_{L} u_{t}^{*}+u_{t}^{*} P_{L} u+v^{*} P_{R} v_{t}+v_{t}^{*} P_{R} v= \\
& =i u^{*}\left(-A_{L}+e_{0, L} e_{0, L}^{T} S_{L}\right) u-\gamma_{L} u^{*} S_{L}^{T} e_{0, L}\left(u_{0}-v_{0}\right)-\tau_{L} u^{*} e_{0, L}\left(\left(S_{L} u\right)_{0}-\left(S_{R} v\right)_{0}\right) \\
& +u^{*} P_{L} \tilde{F}_{L}-i u^{*}\left(-A_{L}^{T}+S_{L}^{T} e_{0, L} e_{0, L}^{T}\right) u-\gamma_{L}^{*}\left(u_{0}^{*}-v_{0}^{*}\right) e_{0, L}^{T} S_{L} u-\tau_{L}^{*}\left(\left(S_{L} u\right)_{0}^{*}-\left(S_{R} v_{0}^{*}\right)\right) e_{0, L}^{T} u \\
& +\tilde{F}_{L}^{*} P_{L} u+i v^{*}\left(-A_{R}-e_{0, R} e_{0, R}^{T} S_{R}\right) v-\gamma_{R} v^{*} S_{R}^{T} e_{0, R}\left(v_{0}-u_{0}\right)-\tau_{R} v^{*} e_{0, R}\left(\left(S_{R} v\right)_{0}-\left(S_{L} u\right)_{0}\right) \\
& +v^{*} P_{R} \tilde{F}_{R}-i v^{*}\left(-A_{R}^{T}-S_{R}^{T} e_{0, R} e_{0, R}^{T}\right) v-\gamma_{R}^{*}\left(v_{0}^{*}-u_{0}^{*}\right) e_{0, R}^{T} S_{R} v-\tau_{R}^{*}\left(\left(S_{R} v\right)_{0}^{*}-\left(S_{L} u\right)_{0}^{*}\right) e_{0, R}^{T} v \\
& +\tilde{F}_{R}^{*} P_{R} v= \\
& =-i u^{*} \underbrace{\left(A_{L}-A_{L}^{T}\right)}_{=0} u-i v^{*} \underbrace{\left(A_{R}-A_{R}^{T}\right)}_{=0} v+i u_{0}^{*}\left(S_{L} u\right)_{0}-i\left(S_{L} u\right)_{0}^{*} u_{0}-\gamma_{L}\left(S_{L} u\right)_{0}^{*}\left(u_{0}-v_{0}\right) \\
& -\tau_{L} u_{0}^{*}\left(\left(S_{L} u\right)_{0}-\left(S_{R} v\right)_{0}\right)-\gamma_{L}^{*}\left(u_{0}^{*}-v_{0}^{*}\right)\left(S_{L} u\right)_{0}-\tau_{L}^{*}\left(\left(S_{L} u\right)_{0}^{*}-\left(S_{R} v\right)_{0}^{*}\right) u_{0}-i v_{0}^{*}\left(S_{R} v\right)_{0} \\
& +i\left(S_{R} v\right)_{0}^{*} v_{0}-\gamma_{R}\left(S_{R} v\right)_{0}^{*}\left(v_{0}-u_{0}\right)-\tau_{R} v_{0}^{*}\left(\left(S_{R} v\right)_{0}-\left(S_{L} u\right)_{0}\right)-\gamma_{R}^{*}\left(v_{0}^{*}-u_{0}^{*}\right)\left(S_{R} v\right)_{0} \\
& -\tau_{R}^{*}\left(\left(S_{R} v\right)_{0}^{*}-\left(S_{L} u\right)_{0}^{*}\right) v_{0}+u^{*} P_{L} \tilde{F}_{L}+\tilde{F}_{L}^{*} P_{L} u+v^{*} P_{R} \tilde{F}_{R}+\tilde{F}_{R}^{*} P_{R} v .
\end{aligned}
$$

Collecting terms leads to the following estimate

$$
\begin{aligned}
& \frac{d}{d t}\|u\|_{P_{L}}^{2}+\frac{d}{d t}\|v\|_{P_{R}}^{2}= \\
& {\left[\begin{array}{c}
u_{0} \\
\left(S_{L} u\right)_{0} \\
v_{0} \\
\left(S_{R} v\right)_{0}
\end{array}\right]^{*} \underbrace{\left[\begin{array}{cccc}
0 & i-\gamma_{L}^{*}-\tau_{L} & 0 & \gamma_{R}^{*}+\tau_{L} \\
-i-\gamma_{L}-\tau_{L}^{*} & 0 & \gamma_{L}+\tau_{R}^{*} & 0 \\
0 & \gamma_{L}^{*}+\tau_{R} & 0 & -i-\gamma_{R}^{*}-\tau_{R} \\
\gamma_{R}+\tau_{L}^{*} & 0 & i-\gamma_{R}-\tau_{R}^{*} & 0
\end{array}\right]}\left[\begin{array}{c}
u_{0} \\
\left(S_{L} u\right)_{0} \\
v_{0} \\
\left(S_{R} v\right)_{0}
\end{array}\right]+} \\
& +u^{*} P_{L} \tilde{F}_{L}+\tilde{F}_{L}^{*} P_{L} u+v^{*} P_{R} \tilde{F}_{R}+\tilde{F}_{R}^{*} P_{R} v .
\end{aligned}
$$

With the penalty parameters (2.7) $\Delta$ vanishes and we get

$$
\begin{array}{r}
\frac{d}{d t}\|u\|_{P_{L}}^{2}+\frac{d}{d t}\|v\|_{P_{R}}^{2}=u^{*} P_{L} \tilde{F}_{L}+\tilde{F}_{L}^{*} P_{L} u+v^{*} P_{R} \tilde{F}_{R}+\tilde{F}_{R}^{*} P_{R} v \\
\leq 2\|u\|_{P_{L}}\left\|\tilde{F}_{L}\right\|_{P_{L}}+2\|v\|_{P_{R}}\left\|\tilde{F}_{R}\right\|_{P_{R}} \leq\|u\|_{P_{L}}^{2}+\left\|\tilde{F}_{L}\right\|_{P_{L}}^{2}+\|v\|_{P_{R}}^{2}+\left\|\tilde{F}_{R}\right\|_{P_{R}}^{2} .
\end{array}
$$

Integrating in time using integrating factor $e^{t}$ yields the desired estimate (2.6).

\subsection{Two dimensions}

For the two-dimensional problem we introduce the following notation:

$$
E_{0, L}=e_{0, L} e_{0, L}^{T}, \quad E_{0, R}=e_{0, R} e_{0, R}^{T}, \quad E_{0, R L}=e_{0, R} e_{0, L}^{T}, \quad E_{0, L R}=e_{0, L} e_{0, R}^{T} .
$$


We introduce the Kronecker product $\otimes$, defined as

$$
A \otimes B=\left[\begin{array}{cccc}
a_{11} B & \ldots & a_{1 n} B & \ldots \\
\vdots & & \vdots & \\
a_{m 1} B & \ldots & a_{m n} B & \ldots \\
\vdots & & \vdots & \ddots
\end{array}\right]
$$

where $A$ and $B$ are operators. The general notation used for expanding an operator in an additional dimension is $\boldsymbol{A}_{\boldsymbol{x}}=A_{x} \otimes I_{y}, \boldsymbol{A}_{\boldsymbol{y}}=I_{x} \otimes A_{y}$. The corresponding semi-discretization of two adjoining nonconforming grid blocks in two space dimensions of $(2.2)$ is

$$
\begin{aligned}
\boldsymbol{u}_{t} & =i\left\{\boldsymbol{Q}_{\boldsymbol{x}_{L}}+\boldsymbol{Q}_{\boldsymbol{y}_{L}}\right\} \boldsymbol{u}-\gamma_{L} \boldsymbol{P}_{\boldsymbol{x}_{L}}^{-\mathbf{1}} \boldsymbol{S}_{\boldsymbol{x}_{L}}^{\boldsymbol{T}}\left(\boldsymbol{E}_{\mathbf{0}, \boldsymbol{x}_{L}} \boldsymbol{u}-\left(E_{0, L R} \otimes I_{F 2 C}\right) \boldsymbol{v}\right) \\
& -\tau_{L} \boldsymbol{P}_{\boldsymbol{x}_{L}}^{-\mathbf{1}}\left(\boldsymbol{E}_{\mathbf{0}, \boldsymbol{x}_{L}} \boldsymbol{S}_{\boldsymbol{x}_{L}} \boldsymbol{u}-\left(E_{0, L R} \otimes I_{F 2 C}\right) \boldsymbol{S}_{\boldsymbol{x}_{R}} \boldsymbol{v}\right)+\tilde{\boldsymbol{F}}_{\boldsymbol{L}}, \\
\boldsymbol{v}_{t} & =i\left\{\boldsymbol{Q}_{\boldsymbol{x}_{\boldsymbol{R}}}+\boldsymbol{Q}_{\boldsymbol{y}_{R}}\right\} \boldsymbol{v}-\gamma_{R} \boldsymbol{P}_{\boldsymbol{x}_{R}}^{-\mathbf{1}} \boldsymbol{S}_{\boldsymbol{x}_{R}}^{\boldsymbol{T}}\left(\boldsymbol{E}_{\mathbf{0}, \boldsymbol{x}_{\boldsymbol{R}}} \boldsymbol{v}-\left(E_{0, R L} \otimes I_{C 2 F}\right) \boldsymbol{u}\right) \\
& -\tau_{R} \boldsymbol{P}_{\boldsymbol{x}_{R}}^{-\mathbf{1}}\left(\boldsymbol{E}_{\mathbf{0}, \boldsymbol{x}_{\boldsymbol{R}}} \boldsymbol{S}_{\boldsymbol{x}_{R}} \boldsymbol{v}-\left(E_{0, R L} \otimes I_{C 2 F}\right) \boldsymbol{S}_{\boldsymbol{x}_{L}} \boldsymbol{u}\right)+\tilde{\boldsymbol{F}}_{\boldsymbol{R}}, \\
& t \geq 0, \boldsymbol{u}(0)=\boldsymbol{f}_{\mathbf{1}}, \boldsymbol{v}(0)=\boldsymbol{f}_{\mathbf{2}},\|\boldsymbol{u}(t)\|_{h}<\infty,\|\boldsymbol{v}(t)\|_{h}<\infty,
\end{aligned}
$$

see figure 2. $I_{F 2 C}$ and $I_{C 2 F}$ are interpolation operators, interpolating from the fine to the coarse grid, and from the coarse to the fine grid, respectively. Suitable block-to-block interpolation operators for nonconforming grid blocks with a grid refinement ratio of $2: 1$ were constructed for SBP operators of different orders of accuracy in [7]. The interpolation operators are, in essence, averaging procedures with the same order of accuracy as the corresponding SBP operators. They satisfy the property

$$
P_{y_{L}} I_{F 2 C}=I_{C 2 F}^{T} P_{y_{R}},
$$

which will be essential in order to obtain a stable interface treatment for the Schrödinger equation.

As discussed in the introduction the $L_{2}$ norm of the continuous solution is preserved. Therefore, we aim at non-decaying energy estimates for the interface problem, as in the stability estimates in [8].

In the analysis we have assumed that the coarse domain is on the left side, as in figure 2 . 


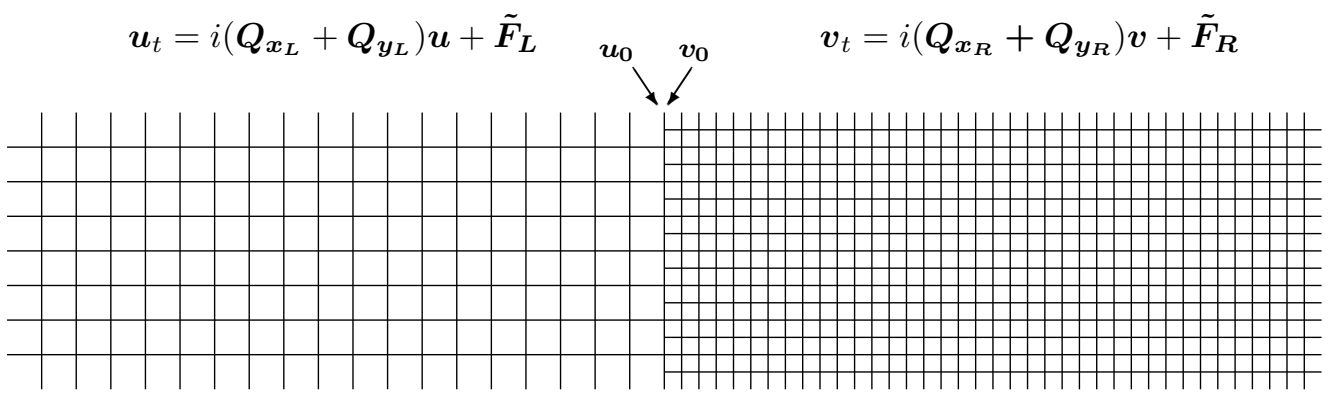

Figure 2: Two adjoining nonconforming grid blocks in two dimensions.

Theorem 2.2. The SBT-SAT interface coupling (2.9), (2.10) with interpolation operators that satisfy (2.11) is stable by the estimate

$\|\boldsymbol{u}(T)\|_{\boldsymbol{P}_{\boldsymbol{L}}}^{2}+\|\boldsymbol{v}(T)\|_{\boldsymbol{P}_{\boldsymbol{R}}}^{2} \leq\left\|\boldsymbol{f}_{\mathbf{1}}\right\|_{\boldsymbol{P}_{\boldsymbol{L}}}^{2}+\left\|\boldsymbol{f}_{\mathbf{2}}\right\|_{\boldsymbol{P}_{\boldsymbol{R}}}^{2}+e^{t_{0}} \int_{0}^{t_{0}}\left\|\tilde{\boldsymbol{F}}_{\boldsymbol{L}}(\tau)\right\|_{\boldsymbol{P}_{\boldsymbol{L}}}^{2}+\left\|\tilde{\boldsymbol{F}}_{\boldsymbol{R}}(\boldsymbol{\tau})\right\|_{\boldsymbol{P}_{\boldsymbol{R}}}^{2} d \tau$,

if the penalty parameters are chosen as

$$
\gamma_{L}=-\frac{i}{2}, \quad \gamma_{R}=\frac{i}{2}, \quad \tau_{L}=\frac{i}{2}, \quad \tau_{R}=-\frac{i}{2} .
$$

Proof: In the two dimensional case energy estimates are obtained by multiplying the two equations with $\boldsymbol{u}^{*} \boldsymbol{P}_{\boldsymbol{L}}$ and $\boldsymbol{v}^{*} \boldsymbol{P}_{\boldsymbol{R}}$, respectively, where $\boldsymbol{P}_{\boldsymbol{L}}=P_{x_{L}} \otimes P_{y_{L}}, \boldsymbol{P}_{\boldsymbol{R}}=P_{x_{R}} \otimes P_{y_{R}}$. We make use of the fact that that the interpolation operators satisfy property $(2.11)$, which leads to the estimate

$$
\begin{aligned}
& \frac{d}{d t}\|\boldsymbol{u}\|_{\boldsymbol{P}_{\boldsymbol{L}}}^{2}+\frac{d}{d t}\|\boldsymbol{v}\|_{\boldsymbol{P}_{\boldsymbol{R}}}^{2}= \\
& {\left[\begin{array}{c}
\boldsymbol{u}_{\mathbf{0}} \\
\left(\boldsymbol{S}_{\boldsymbol{x}_{\boldsymbol{L}}} \boldsymbol{u}\right)_{\mathbf{0}} \\
\boldsymbol{v}_{\mathbf{0}} \\
\left(\boldsymbol{S}_{\boldsymbol{x}_{\boldsymbol{R}}} \boldsymbol{v}\right)_{\mathbf{0}}
\end{array}\right]^{P_{y_{L}}}\left[\begin{array}{cccc}
0 & i-\gamma_{L}^{*}-\tau_{L} & 0 & \gamma_{R}^{*}+\tau_{L} \\
-i-\gamma_{L}-\tau_{L}^{*} & 0 & \gamma_{L}+\tau_{R}^{*} & 0 \\
0 & \gamma_{L}^{*}+\tau_{R} & 0 & -i-\gamma_{R}^{*}-\tau_{R} \\
\gamma_{R}+\tau_{L}^{*} & 0 & i-\gamma_{R}-\tau_{R}^{*} & 0
\end{array}\right] \text { 。 }} \\
& {\left[\begin{array}{cccc}
P_{y_{L}} & P_{y_{L}} & P_{y_{L}} I_{F 2 C} & P_{y_{L}} I_{F 2 C} \\
P_{y_{L}} & P_{y_{L}} & P_{y_{L}} I_{F 2 C} & P_{y_{L}} I_{F 2 C} \\
P_{y_{R}} I_{C 2 F} & P_{y_{R}} I_{C 2 F} & P_{y_{R}} & P_{y_{R}} \\
P_{y_{R}} I_{C 2 F} & P_{y_{R}} I_{C 2 F} & P_{y_{R}} & P_{y_{R}}
\end{array}\right]\left[\begin{array}{c}
\boldsymbol{u}_{\mathbf{0}} \\
\left(\boldsymbol{S}_{\boldsymbol{x}_{\boldsymbol{L}}} \boldsymbol{u}\right)_{\mathbf{0}} \\
\boldsymbol{v}_{\mathbf{0}} \\
\left(\boldsymbol{S}_{\boldsymbol{x}_{R}} \boldsymbol{v}\right)_{\mathbf{0}}
\end{array}\right]} \\
& +\boldsymbol{u}^{*} \boldsymbol{P}_{\boldsymbol{L}} \tilde{\boldsymbol{F}}_{\boldsymbol{L}}+\tilde{\boldsymbol{F}}_{L}^{*} \boldsymbol{P}_{\boldsymbol{L}} \boldsymbol{u}+\boldsymbol{v}^{*} \boldsymbol{P}_{\boldsymbol{R}} \tilde{\boldsymbol{F}}_{\boldsymbol{R}}+\tilde{\boldsymbol{F}}_{\boldsymbol{R}}^{*} \boldsymbol{P}_{\boldsymbol{R}} \boldsymbol{v},
\end{aligned}
$$

where $\circ$ denotes an Hadamard product. As in the one-dimensional case choosing the penalty parameters as in (2.13), and integrating in time with integrating factor $e^{t}$ leads to (2.12). 
We note that with this choice of penalty parameters the homogeneous versions of the interface problems (2.4), (2.5) and (2.9), (2.10) lead to discrete energy preservation. This is desirable, since the $L_{2}$ norm is preserved for the continuous problem.

\subsection{Multiple dimensions}

We also consider the multi-block discretization in $N$ dimensions, where $N$ is an arbitrary number. We use the notation $\boldsymbol{A}_{\mathbf{1}}=A_{1} \otimes I_{2} \otimes \cdots \otimes I_{N}, \boldsymbol{A}_{\mathbf{2}}=$ $I_{1} \otimes A_{2} \otimes \cdots \otimes I_{N}$, and so on, to expand operators in higher dimensions. $I_{q}$ is the identity operator corresponding to dimension $q$. The interface will be an $N$-1-dimensional hypersurface and we again assume that the fine/coarse grid size ratio across the interface is $2: 1$. The semi-discretization in $N$ dimensions is

$$
\begin{aligned}
& \boldsymbol{u}_{t}=i\left\{\boldsymbol{Q}_{\mathbf{1}, \boldsymbol{L}}+\boldsymbol{Q}_{\mathbf{2}, \boldsymbol{L}}+\cdots+\boldsymbol{Q}_{\boldsymbol{N}, \boldsymbol{L}}\right\} \boldsymbol{u} \\
& -\gamma_{L} \boldsymbol{P}_{\mathbf{1 , L}}^{-\mathbf{1}} \boldsymbol{S}_{\mathbf{1}, \boldsymbol{L}}^{\boldsymbol{T}}\left(\boldsymbol{E}_{\mathbf{0}, \mathbf{1}, \boldsymbol{L}} \boldsymbol{u}-\left(E_{0, L R} \otimes I_{F 2 C, 2} \otimes \cdots \otimes I_{F 2 C, N}\right) \boldsymbol{v}\right) \\
& -\tau_{L} \boldsymbol{P}_{\mathbf{1 , L}}^{-\mathbf{1}}\left(\boldsymbol{E}_{\mathbf{0 , 1 , L}} \boldsymbol{S}_{\mathbf{1}, \boldsymbol{L}} \boldsymbol{u}-\left(E_{0, L R} \otimes I_{F 2 C, 2} \otimes \cdots \otimes I_{F 2 C, N}\right) \boldsymbol{S}_{\mathbf{1}, \boldsymbol{R}} \boldsymbol{v}\right)+\tilde{\boldsymbol{F}}_{\boldsymbol{L}}, \\
& \boldsymbol{v}_{t}=i\left\{\boldsymbol{Q}_{\mathbf{1}, \boldsymbol{R}}+\boldsymbol{Q}_{\mathbf{2}, \boldsymbol{R}}+\cdots+\boldsymbol{Q}_{\boldsymbol{N}, \boldsymbol{R}}\right\} \boldsymbol{v} \\
& -\gamma_{R} \boldsymbol{P}_{\mathbf{1}, \boldsymbol{R}}^{-\mathbf{1}} \boldsymbol{S}_{\mathbf{1}, \boldsymbol{R}}^{\boldsymbol{T}}\left(\boldsymbol{E}_{\mathbf{0}, \mathbf{1}, \boldsymbol{R}} \boldsymbol{v}-\left(E_{0, R L} \otimes I_{C 2 F, 2} \otimes \cdots \otimes I_{C 2 F, N}\right) \boldsymbol{u}\right) \\
& \text { - } \tau_{R} \boldsymbol{P}_{\mathbf{1 , R}}^{-\mathbf{1}}\left(\boldsymbol{E}_{\mathbf{0 , 1}, \boldsymbol{R}} \boldsymbol{S}_{\mathbf{1}, \boldsymbol{R}} \boldsymbol{v}-\left(E_{0, R L} \otimes I_{C 2 F, 2} \otimes \cdots \otimes I_{C 2 F, N}\right) \boldsymbol{S}_{\mathbf{1}, L} \boldsymbol{u}\right)+\tilde{\boldsymbol{F}}_{\boldsymbol{R}}, \\
& t \geq 0, \boldsymbol{u}(0)=\boldsymbol{f}_{\mathbf{1}}, \boldsymbol{v}(0)=\boldsymbol{f}_{\mathbf{2}},\|\boldsymbol{u}(t)\|_{h}<\infty,\|\boldsymbol{v}(t)\|_{h}<\infty .
\end{aligned}
$$

We can show that the SBT-SAT interface coupling (2.14), (2.15) is stable by theorem 2.2. The proof is analogous to the two-dimensional case. We multiply equations (2.14) and (2.15), from the left with $\boldsymbol{u}^{*} \boldsymbol{P}_{\boldsymbol{L}}$ and $\boldsymbol{v}^{*} \boldsymbol{P}_{\boldsymbol{R}}$, respectively, where $\boldsymbol{P}_{\boldsymbol{L}}=P_{1, L} \otimes P_{2, L} \otimes \cdots \otimes P_{N, L}, \boldsymbol{P}_{\boldsymbol{R}}=$ $P_{1, R} \otimes P_{2, R} \otimes \cdots \otimes P_{N, R}$.

We now choose the same penalty parameters as in the one- and twodimensional cases ( 2.13) and integrate in time with integration factor $e^{t}$. If

$$
P_{q, L} I_{F 2 C, q}=I_{C 2 F, q}^{T} P_{q, R}, q=2, \ldots N,
$$

we obtain (2.12). Note that the interpolation operators from [7] satisfy (2.16) by construction. 


\section{Accuracy at the grid interface}

We will use the stability estimate (2.6) derived in section 2 to analyse the accuracy of the approximation in one dimension. A schematic summary of the accuracy analysis procedure is given below. For a more thorough description of the methodology we refer to [8], where we presented a framework for analysing the accuracy of stable boundary closures.

1. We consider the pointwise error, defined as the difference between the analytic solution of (2.1) and the numerical solution of (2.4) and (2.5). The error is partitioned into an interior error, arising from the truncation error in the interior, and a boundary error arising from the lower order truncation error on both sides of the interface.

2. Assuming that the semi-discretization is stable, the interior error can directly be estimated through the energy estimate (2.6). The convergence rate in the estimate will be of the same order as that of the interior scheme.

3. The boundary error is estimated using normal mode analysis, and will, because of the lower order boundary truncation error, generally determine the global convergence rate. The modified stencils in the vicinity of the interface give rise to a system $C(\tilde{s}) \Sigma=\hat{T}_{B}$, where $\Sigma$ is the vector of coefficients that should be determined, $\hat{T}_{B}$ depends on the local truncation error, $s$ is the dual variable in time, and $\tilde{s}=s h^{2}$.

4. We are looking for an asymptotic accuracy result, and consider $s$ bounded and $h$ sufficiently small. In particular, if $C(0)$ is non-singular $\Sigma$ can be determined directly in terms of the inverse of $C(0)$, as was the case in [8]. Here, $C(0)$ will be singular and we need to consider $C(\tilde{s})$ in a vicinity of $\tilde{s}=0$ in order to get a pointwise error estimate in Laplace space.

5. Error estimates in physical space can be derived using a Parseval equality.

Consider (2.1) and the corresponding discretization constituted by (2.4) and (2.5). The error vector for the pointwise error on the coarser grid (on the left side) is denoted $\tilde{\varepsilon}^{L}, \tilde{\varepsilon}_{j}^{L}(t)=U\left(x_{j}, t\right)-u_{j}(t), j=0,-1,-2, \ldots$, and the error on the fine grid $\tilde{\varepsilon}^{R}, \tilde{\varepsilon}_{i}^{R}(t)=U\left(x_{i}, t\right)-v_{i}(t), i=0,1,2, \ldots$ Assuming that the continuous problem has a smooth solution $U(x, t)$, we 


$$
\begin{aligned}
& \text { get } \\
& \tilde{\varepsilon}_{t}^{L}=i Q_{L} \tilde{\varepsilon}^{L}-\gamma_{L} P_{L}^{-1} S_{L}^{T} e_{0, L}\left(\tilde{\varepsilon}_{0}^{L}-\tilde{\varepsilon}_{0}^{R}\right)-\tau_{L} P_{L}^{-1} e_{0, L}\left(\left(S_{L} \tilde{\varepsilon}^{L}\right)_{0}-\left(S_{R} \tilde{\varepsilon}^{R}\right)_{0}\right)+\tilde{T}_{L}, \\
& \tilde{\varepsilon}_{t}^{R}=i Q_{R} \tilde{\varepsilon}^{R}-\gamma_{R} P_{R}^{-1} S_{R}^{T} e_{0, R}\left(\tilde{\varepsilon}_{0}^{R}-\tilde{\varepsilon}_{0}^{L}\right)-\tau_{R} P_{R}^{-1} e_{0, R}\left(\left(S_{R} \tilde{\varepsilon}^{R}\right)_{0}-\left(S_{L} \tilde{\varepsilon}^{L}\right)_{0}\right)+\tilde{T}_{R} .
\end{aligned}
$$

The $p$ components of both $\tilde{T}_{R}$ and $\tilde{T}_{L}$ that are closest to the interface are of order $\mathcal{O}\left(h^{m}\right)$ while all other components of these truncation errors are of order $\mathcal{O}\left(h^{2 m}\right)$. We denote these parts by $T_{R / L}$ and $T_{R, L}^{I}$, respectively. Then,

$$
\tilde{T}_{R / L}=h^{m} T_{R / L}+h^{2 m} T_{R / L}^{I}, \quad T_{i} \equiv 0,|i| \geq p, \quad T_{i}^{I} \equiv 0,|i|<p .
$$

Theorem 3.1. Consider (2.4), (2.5) for $m=1,2$ and assume the continuous solution is smooth, with sufficiently many derivatives bounded in $\|\cdot\|_{h}$ and $|\cdot|_{\infty}$ norm in all intervals $0 \leq t \leq t_{0}$. The error is partitioned similarly to $T^{B}$ and $T^{I}$, into $\varepsilon^{L}, \varepsilon^{L, I}, \varepsilon^{R}$ and $\varepsilon^{R, I}$. It satisfies

$$
\begin{aligned}
\sqrt{\left\|\varepsilon^{L, I, m}(t)\right\|_{h}^{2}+\left\|\varepsilon^{R, I, m}(t)\right\|_{h}^{2}} & \leq h^{2 m} K_{1}^{m}\left(t_{0}\right), \\
\sqrt{\int_{0}^{t_{0}}\left|\varepsilon_{j}^{L, m}(t)\right|^{2}+\left|\varepsilon_{j}^{R, m}(t)\right|^{2} d t} & \leq h^{2 m} K_{2}^{m}\left(t_{0}\right), \\
\sqrt{\int_{0}^{t_{0}}\left\|\varepsilon^{L, m}(t)\right\|_{h}^{2}+\left\|\varepsilon^{R, m}(t)\right\|_{h}^{2} d t} & \leq h^{2 m} K_{3}^{m}\left(t_{0}\right),
\end{aligned}
$$

for $m=1,2$. Here $K_{1}, K_{2}, K_{3}$ depend only on $U$ and some of its derivatives, and on the time interval.

It is easy to estimate $\varepsilon^{L, I}$ and $\varepsilon^{R, I}$ using the energy estimate given in Theorem 2.1.

To derive the estimate for the remaining error we perform a Laplace transformation in time, and consider $\operatorname{Re}(s)>0$. We want to derive an estimate valid in the vicinity of $\tilde{s}=0$. Especially, it must be uniform with respect to $\operatorname{Re}(s) \rightarrow 0$. The $p$ components of $\varepsilon^{L}$ and $\varepsilon^{R}$ closest to the interface have truncation errors of order $m$. These rows as well as the rows that are affected by the penalty parameters need to be included in the system of error equations that are used to derive $C(\tilde{s}) \Sigma=h^{m+2} \hat{F}^{B}$. For orders 2 and 4 , the number of rows in the system is $6(3+3)$ and $8(4+4)$.

Sufficiently far away from the boundary, we have the central finite difference discretizations given by

$$
\begin{aligned}
& m=1: \quad s \hat{\varepsilon}_{k}=i D_{+} D_{-} \hat{\varepsilon}_{k}, \\
& m=2: \quad s \hat{\varepsilon}_{k}=i\left(D_{+} D_{-}-\frac{h^{2}}{12}\left(D_{+} D_{-}\right)^{2}\right) \hat{\varepsilon}_{k},
\end{aligned}
$$


where the operators $D_{+}, D_{-}$are defined as

$$
D_{+} u_{j}=\frac{u_{j+1}-u_{j}}{h}, \quad D_{-} u_{j}=\frac{u_{j}-u_{j-1}}{h},
$$

where $D_{+} D_{-}$is the standard central second order stencil approximating the second derivative. We will need the lemma and the corresponding collorary on characteristic roots from [8, lemma 4.2, corollary 4.3], for the proof of theorem 3.1, and restate them below.

Lemma 3.2. Let $\tilde{s}=s h^{2}$ and consider the characteristic equations corresponding to (3.7) and (3.8). As long as $\operatorname{Re}(\tilde{s})>0$ there are no characteristic roots with $|\kappa|=1$. For $m=1,2$ the admissible roots, satisfying $|\kappa|<1$ for $\operatorname{Re}(\tilde{s})>0$, are

$$
\begin{array}{ll}
m=1: & \kappa_{1}=1+\frac{-1+i}{\sqrt{2}} \sqrt{\tilde{s}}+\mathcal{O}(\tilde{s}), \\
m=2: & \kappa_{1}=1-\frac{1+i}{\sqrt{2}} \sqrt{\tilde{s}}+\mathcal{O}(\tilde{s}), \kappa_{2}=7-4 \sqrt{3}+\mathcal{O}(\tilde{s}),
\end{array}
$$

Corollary 3.3. Consider $|s| \leq K, \operatorname{Re}(s) \geq \eta>0$. For $m=1,2$ the admissible roots $\kappa_{1}$ satisfy

$$
1-\left|\kappa_{1}(\tilde{s})\right|^{2} \geq \frac{\eta}{K} \sqrt{|\tilde{s}|}(1+\sqrt{|\tilde{s}|})
$$

\subsection{Proof of theorem 3.1 for interior order 2}

To begin with we consider (3.1) and (3.2) with only the interior truncation error as forcing. Now, (3.4) follows by applying theorem 2.1.

For the remaining part of the proof, we shall consider the Laplace transform of (3.1) and (3.2), with $\tilde{\varepsilon}^{L}, \tilde{\varepsilon}^{R}$ replaced by $\varepsilon^{L}, \varepsilon^{R}$ and only the interface truncation error as forcing. Equation (3.7) is valid for $\hat{\varepsilon}_{-j}^{L}, \hat{\varepsilon}_{i}^{R}, i, j \geq 4$.

Multiplying the last three rows of (3.1) with $h_{L}^{2}$ and the first three rows of (3.2) with $h_{R}^{2}$, respectively, yields the system

$$
\left[\begin{array}{c}
\tilde{s}_{L} \hat{\varepsilon}_{-2}^{L} \\
\tilde{s}_{L} \hat{\varepsilon}_{-1}^{L} \\
\tilde{s}_{L} \hat{\varepsilon}_{0}^{L} \\
\tilde{s}_{R} \hat{\varepsilon}_{0}^{R} \\
\tilde{s}_{R} \hat{\varepsilon}_{1}^{R} \\
\tilde{s}_{R} \hat{\varepsilon}_{2}^{R}
\end{array}\right]=i\left[\begin{array}{ll}
\tilde{Q}_{L, B} & Q_{L R} \\
Q_{R L} & \tilde{Q}_{R, T}
\end{array}\right]\left[\begin{array}{c}
\hat{\varepsilon}_{-3}^{L} \\
\hat{\varepsilon}_{-2}^{L} \\
\hat{\varepsilon}_{-1}^{L} \\
\hat{\varepsilon}_{0}^{L} \\
\hat{\varepsilon}_{0}^{R} \\
\hat{\varepsilon}_{1}^{R} \\
\hat{\varepsilon}_{2}^{R} \\
\hat{\varepsilon}_{3}^{R}
\end{array}\right]+i\left[\begin{array}{c}
0 \\
0 \\
h_{L}^{3} \hat{F}_{\varepsilon^{L}}^{B} \\
h_{R}^{3} \hat{F}_{\varepsilon^{R}}^{B} \\
0 \\
0
\end{array}\right],
$$


where $\tilde{s}_{L}=s h_{L}^{2}, \tilde{s}_{R}=s h_{R}^{2}=\frac{\tilde{s}_{L}}{4}$, and $\hat{F}_{\varepsilon^{L}}^{B}=\frac{3}{4} \hat{U}_{x x x}, \hat{F}_{\varepsilon^{R}}^{B}=-2 \hat{U}_{x x x}$. For later reference we note that by using the equation we can express these terms as $\hat{F}_{\varepsilon^{L}}^{B}=s \hat{\tilde{F}}_{\varepsilon^{L}}^{B}, \hat{F}_{\varepsilon^{R}}^{B}=s \hat{\tilde{F}}_{\varepsilon^{R}}$, where $\hat{\tilde{F}}_{\varepsilon^{L}}^{B}, \hat{\tilde{F}}_{\varepsilon^{R}}^{B} \sim \hat{U}_{x}$.

Here, $\tilde{Q}_{L, B}$ and $\tilde{Q}_{R, T}$ are $3 \times 4$-matrices. $\tilde{Q}_{L, B}$ consists of $Q_{L, B}$ (defined in section 2.1) extended to three rows to include the penalty terms corresponding to $\hat{\varepsilon}^{L}$ in (3.1). Correspondingly, $\tilde{Q}_{R, T}$ includes $Q_{R, T}$ and the penalty terms corresponding to $\hat{\varepsilon}^{R}$ in (3.2). The coupling across the grid interface is reflected in both $Q_{L R}$ and $Q_{R L}$, which contain the penalty terms corresponding to $\hat{\varepsilon}^{R}$ in (3.1) and vice versa.

For accuracy, we consider the vicinity of $\tilde{s}_{L}=0$. For the interior scheme, we have one admissible root on each side,

$$
\begin{aligned}
& \kappa_{L}\left(\tilde{s}_{L}\right)=1+\sqrt{\tilde{s}_{L} / 2}(-1+i)+\mathcal{O}\left(\tilde{s}_{L}\right), \\
& \kappa_{R}\left(\tilde{s}_{L}\right)=1+\frac{\sqrt{\tilde{s}_{L} / 2}}{2}(-1+i)+\mathcal{O}\left(\tilde{s}_{L}\right) .
\end{aligned}
$$

Thus, the solution has the form

$$
\begin{aligned}
\hat{\varepsilon}_{-j}^{L}=\sigma_{L} \kappa_{L}^{j-2}, & j=2,3, \ldots \\
\hat{\varepsilon}_{i}^{R}=\sigma_{R} \kappa_{R}^{i-2}, & i=2,3, \ldots
\end{aligned}
$$

$\sigma_{L}$ and $\sigma_{R}$ are unknowns to be determined together with the unknowns $\hat{\varepsilon}_{0}^{L}, \hat{\varepsilon}_{-1}^{L}, \hat{\varepsilon}_{0}^{R}$ and $\hat{\varepsilon}_{1}^{R}$. By inserting (3.15), (3.16) into (3.12) above, we get the system

$$
C\left(\tilde{s}_{L}\right) \Sigma=B
$$

where

$$
B=h_{L}^{3}\left[\begin{array}{c}
0 \\
0 \\
-\frac{3}{4} \\
\frac{1}{4} \\
0 \\
0
\end{array}\right] \hat{U}_{x x x}, \quad \Sigma=\left[\begin{array}{c}
\sigma_{L} \\
\sigma_{R} \\
\varepsilon_{-1}^{L} \\
\varepsilon_{0}^{L} \\
\varepsilon_{0}^{R} \\
\varepsilon_{1}^{R}
\end{array}\right]
$$




$$
\begin{aligned}
& C\left(\tilde{s}_{L}\right)=\left[\begin{array}{cccccc}
-i \tilde{s}_{L}+2-\kappa_{L} & 0 & -1 & -\frac{1}{4} & \frac{1}{4} & 0 \\
-1 & 0 & -i \tilde{s}_{L}+2 & 0 & -1 & 0 \\
-\frac{1}{2} & 1 & 0 & -i \tilde{s}_{L}-1 & \frac{9}{2} & -4 \\
\frac{1}{4} & -\frac{1}{2} & -1 & \frac{9}{4} & -\frac{i}{4} \tilde{s}_{L}-1 & 0 \\
0 & -1 & 0 & -1 & 0 & -\frac{i}{4} \tilde{s}_{L}+2 \\
0 & -\frac{i}{4} \tilde{s}_{L}+2-\kappa_{R} & 0 & \frac{1}{4} & -\frac{1}{4} & -1
\end{array}\right]= \\
& =\underbrace{\left[\begin{array}{cccccc}
1 & 0 & -1 & -\frac{1}{4} & \frac{1}{4} & 0 \\
-1 & 0 & 2 & 0 & -1 & 0 \\
-\frac{1}{2} & 1 & 0 & -1 & \frac{9}{2} & -4 \\
\frac{1}{4} & -\frac{1}{2} & -1 & \frac{9}{4} & -1 & 0 \\
0 & -1 & 0 & -1 & 0 & 2 \\
0 & 1 & 0 & \frac{1}{4} & -\frac{1}{4} & -1
\end{array}\right]}_{C(0)}+\sqrt{\tilde{s}_{L}} \frac{1}{\sqrt{2}}(-1+i)\left[\begin{array}{cccccc}
-1 & 0 & 0 & 0 & 0 & 0 \\
0 & 0 & 0 & 0 & 0 & 0 \\
0 & 0 & 0 & 0 & 0 & 0 \\
0 & 0 & 0 & 0 & 0 & 0 \\
0 & 0 & 0 & 0 & 0 & 0 \\
0 & -\frac{1}{2} & 0 & 0 & 0 & 0
\end{array}\right]+\mathcal{O}\left(\tilde{s}_{L}\right) .
\end{aligned}
$$

Here, $\mathrm{C}(0)$ is singular with rank 5 . To investigate accuracy we expand $\mathrm{C}\left(\tilde{s}_{L}\right)$ in the vicinity of $\tilde{s}_{L}=0$ by using the Taylor expansions of $\kappa_{L}$ and $\kappa_{R}$ that are given by (3.13) and (3.14). We now have the following lemma.

Lemma 3.4. Consider the $n \times n$ linear system $(A+\delta E) x=b$ where $A$ is singular with rank $n-1$. Let $U S V^{*}=A$ be the singular value decomposition of $A$. If $\left(U^{*} E V\right)_{n n} \neq 0$ then for all sufficiently small $|\delta|$ we get

$$
\left|(A+\delta E)^{-1}\right| \leq\left(2 \delta\left(U^{*} E V\right)_{n n}\right)^{-1} .
$$

If, in addition, $b$ is in the column space of $C(0)$ then for all sufficiently small $|\delta|$, we have

$$
|x| \leq c|b|
$$

Here $c$ is independent of $\delta$.

Here, $|v|$ and $|A|$ denote the usual Euclidian vector norm and associated matrix norm, respectively.

Proof: by construction. The linear system can equivalently be written as

$$
\left(S+\delta U^{*} E V\right) \tilde{x}=\tilde{b}, \quad \tilde{x}=V^{*} x, \quad \tilde{b}=U^{*} b .
$$

The lower triangular elements can be eliminated, corresponding to multiplication by the inverse of a lower triangular matrix $L=I+\mathcal{O}(\delta)$. The remaining upper triangular matrix $U$ has diagonal elements

$$
U_{i i}=S_{i i}+O(\delta), i=1, . . n-1, U_{n n}=\delta\left(U^{*} E V\right)_{n n}+O\left(\delta^{2}\right) .
$$

The elements above the diagonal are all $O(\delta)$. Thus the elimination can be continued without introducing any singular dependance on $\delta$, proving the 
first claim. To prove the second claim we note that the last element of $\tilde{b}$ vanishes and hence $\left(L^{-1} \tilde{b}\right)_{n}=O(\delta)$. Consequently, the last element of $\tilde{x}$ is bounded independently of $\delta$. The back-substitution can proceed as in the first case, proving the second claim.

In our case we compute the SVD for $C(0)$, obtaining $\left(U^{*} E V\right)_{66}=$ $0.1721(1-i) \neq 0$ and observe that the right hand side is not in the column space. We use the first bound given by lemma 3.4, (3.19) with $\delta=\sqrt{\left|\tilde{s}_{L}\right|}$. Thus we have point wise bounds, valid in a neighborhood of $\tilde{s}_{L}=0$. Remember that $|s| \leq K$ !

$\left|\hat{\varepsilon}_{j}^{L}\right|+\left|\hat{\varepsilon}_{j}^{R}\right| \leq h^{3} \frac{C}{\sqrt{\left|\tilde{s}_{L}\right|}}|s|\left(\left|\hat{\tilde{F}}_{\varepsilon^{L}}\right|+\left|\hat{\tilde{F}}_{\varepsilon^{R}}\right|\right) \leq h^{2} \tilde{C}\left(\left|\hat{\tilde{F}}_{\varepsilon^{L}}\right|+\left|\hat{\tilde{F}}_{\varepsilon^{R}}\right|\right), \quad j=0,1, \ldots$

By Parsevals inequality we have

$$
\int_{0}^{t_{0}}\left(\left|\varepsilon_{j}^{L}(t)\right|^{2}+\left|\varepsilon_{j}^{R}(t)\right|^{2}\right) d t \leq h^{4} K\left(t_{0}\right), \quad j=0,1, \ldots,
$$

proving (3.5) for $m=1$, since $K\left(t_{0}\right) \sim \int_{0}^{t_{0}}\left|U_{x}(0, t)\right| d t$ depends only on the continuous solution. Since the $\kappa^{\prime}$ s are the same as in [8], we can use the first inequality in (3.23) together with (3.11) to obtain

$$
\begin{aligned}
&\left\|\hat{\varepsilon}^{L}\right\|_{h}^{2}+\left\|\hat{\varepsilon}^{R}\right\|_{h}^{2}= \\
&=\sigma_{L}^{2} \sum_{0}^{\infty} h\left|\kappa_{L}\left(\tilde{s}_{L}\right)\right|^{2}+\sigma_{R}^{2} \sum_{0}^{\infty} h\left|\kappa_{R}\left(\tilde{s}_{R}\right)\right|^{2} \\
&=\frac{h \sigma_{L}^{2}}{1-\left|\kappa_{L}\left(\tilde{s}_{L}\right)\right|^{2}}+\frac{h \sigma_{R}^{2}}{1-\left|\kappa_{R}\left(\tilde{s}_{R}\right)\right|^{2}} \\
& \leq K \frac{h^{7}|s|^{2}}{\left|\tilde{s}_{L}\right| \eta \sqrt{\left|\tilde{s}_{L}\right|}}-\max _{L} \leq x \leq h_{R} \\
&\left|\hat{U}_{x}(x, s)\right|^{2} \\
& \leq\left. h^{4} \frac{K}{\eta} \max _{L}\right|_{h_{L} \leq h_{R}}\left|\hat{U}_{x}(x, s)\right|^{2},
\end{aligned}
$$

for any $\eta>0$. We can now derive (3.6), noting that the interface error is of order 2 .

\subsection{Proof of theorem 3.1 for interior order 4}

As in the previous case, we directly obtain (3.4) from 2.1. For the remaining estimates we consider the Laplace transform of (3.1) and (3.2), multiplied with $h_{L}^{2}$ and $h_{R}^{2}$, respectively. Taking the penalty terms and 
specific coefficients into account we get the system

$$
\left[\begin{array}{c}
\tilde{s}_{L} \hat{\varepsilon}_{-3}^{L} \\
\tilde{s}_{L} \hat{\varepsilon}_{-2}^{L} \\
\tilde{s}_{L} \hat{\varepsilon}_{-1}^{L} \\
\tilde{s}_{L} \hat{\varepsilon}_{0}^{L} \\
\tilde{s}_{R} \hat{\varepsilon}_{0}^{R} \\
\tilde{s}_{R} \hat{\varepsilon}_{1}^{R} \\
\tilde{s}_{R} \hat{\varepsilon}_{2}^{R} \\
\tilde{s}_{R} \hat{\varepsilon}_{3}^{R}
\end{array}\right]=i\left[\begin{array}{c}
\tilde{Q}_{L, B} \\
Q_{R L}
\end{array}\right.
$$

$$
\left.\hat{Q}_{L R}\right]\left[\begin{array}{c}
\hat{\varepsilon}_{-T}^{L} \\
\hat{\varepsilon}_{-4}^{L} \\
\hat{\varepsilon}_{-3}^{L} \\
\hat{\varepsilon}_{-}^{L} \\
\hat{\varepsilon}_{-1}^{L} \\
\hat{\varepsilon}_{0}^{L} \\
\hat{\varepsilon}_{0}^{R} \\
\hat{\varepsilon}_{1}^{R} \\
\hat{\varepsilon}_{2}^{R} \\
\hat{\varepsilon}_{3}^{R} \\
\hat{\varepsilon}_{4}^{R} \\
\hat{\varepsilon}_{5}^{R}
\end{array}\right]+i\left[\begin{array}{c}
h_{L}^{4} \hat{F}_{B, \varepsilon^{L}}^{-3} \\
h_{L}^{4} \hat{F}_{B, \varepsilon^{L}}^{-2} \\
h_{L}^{4} \hat{F}_{B, 1}^{-1} \\
h_{L}^{4} \hat{F}_{B, \varepsilon^{L}}^{0} \\
h_{R}^{4} \hat{F}_{B, \varepsilon^{R}}^{0} \\
h_{R}^{4} \hat{F}_{B, \varepsilon^{R}}^{1} \\
h_{R}^{4} \hat{F}_{B, \varepsilon^{R}}^{2} \\
h_{R}^{4} \hat{F}_{B, \varepsilon^{R}}^{3}
\end{array}\right] \text {, }
$$

where $\tilde{Q}_{L, B}, \tilde{Q}_{R, T}$ constitute $Q_{L, B}, Q_{R, T}$ and corresponding penalty terms, and $Q_{L R}, Q_{R L}$ constitute only penalty terms, as defined in the previous section. Here, $\tilde{Q}_{L, B}$ and $\tilde{Q}_{R, T}$ are of dimension $4 \times 6$, as are $Q_{L, B}$ and $Q_{R, T}$.

In the fourth order case there are two admissible roots, $\kappa_{1}$ and $\kappa_{2}$. Thus, we make the ansatz

$$
\begin{aligned}
\hat{\varepsilon}_{-j}^{L} & =\sigma_{L, 1} \kappa_{L, 1}^{j-2}+\sigma_{L, 2} \kappa_{L, 2}^{j-2}, & & j=2,3, \ldots, \\
\hat{\varepsilon}_{i}^{R} & =\sigma_{R, 1} \kappa_{R, 1}^{i-2}+\sigma_{R, 2} \kappa_{R, 2}^{i-2}, & & i=2,3, \ldots
\end{aligned}
$$

We follow the same procedure as in the second order case and set up the system (3.17) by inserting (3.27) and (3.28) into (3.26). In this case we have $\Sigma=\left[\sigma_{L, 1} \sigma_{L, 2} \sigma_{R, 1} \sigma_{R, 2} \hat{\varepsilon}_{-1}^{L} \hat{\varepsilon}_{0}^{L} \hat{\varepsilon}_{0}^{R} \varepsilon_{1}^{R}\right]^{T}$.

Expanding the truncation error around $x=0$ and discarding higher order terms leads to the following right hand side in equation (3.17),

$$
B=h_{L}^{4}\left[\begin{array}{c}
\frac{11}{588} \\
\frac{5}{516} \\
-\frac{1}{12} \\
\frac{53}{102} \\
-\frac{461}{3264} \\
-\frac{1}{192} \\
\frac{5}{8256} \\
\frac{11}{9408}
\end{array}\right] \hat{U}_{x x x x}(0, t) .
$$


The admissible roots in the interior are given by (3.10) and we get

$$
\begin{aligned}
& C\left(\tilde{s}_{L}\right)=\underbrace{C(0)}_{\left.\begin{array}{cccccccc}
-\frac{1}{49} & \frac{-37-8 \sqrt{3}}{49} & 0 & 0 & 0 & \frac{9}{49} & -\frac{8}{49} & 0 \\
\frac{55}{43} & \frac{85+12 \sqrt{3}}{43} & 0 & 0 & -\frac{59}{43} & -\frac{32}{43} & \frac{36}{43} & 0 \\
-1 & -1 & 0 & 0 & 2 & \frac{13}{59} & -\frac{72}{59} & 0 \\
-\frac{23}{17} & \frac{31-36 \sqrt{3}}{17} & \frac{56}{17} & \frac{-40+64 \sqrt{3}}{17} & \frac{13}{17} & -2 & \frac{132}{17} & -\frac{144}{17} \\
\frac{14}{17} & \frac{-10+16 \sqrt{3}}{17} & -\frac{23}{17} & \frac{31-36 \sqrt{3}}{17} & -\frac{36}{17} & \frac{66}{17} & -2 & \frac{13}{17} \\
0 & 0 & -1 & -1 & 0 & -\frac{72}{59} & \frac{13}{59} & 2 \\
0 & 0 & \frac{55}{43} & \frac{85+12 \sqrt{3}}{43} & 0 & \frac{36}{43} & -\frac{32}{43} & -\frac{59}{43} \\
0 & 0 & -\frac{1}{49} & \frac{-37-8 \sqrt{3}}{49} & 0 & -\frac{8}{49} & \frac{9}{49} & 0
\end{array}\right]} \\
& -\sqrt{\tilde{s}_{L}} \frac{1}{\sqrt{2}}(1+i)\left[\begin{array}{cccccccc}
\frac{2}{49} & 0 & 0 & 0 & 0 & 0 & 0 & 0 \\
-\frac{51}{43} & 0 & 0 & 0 & 0 & 0 & 0 & 0 \\
0 & 0 & 0 & 0 & 0 & 0 & 0 & 0 \\
\frac{9}{17} & 0 & -\frac{8}{17} & 0 & 0 & 0 & 0 & 0 \\
-\frac{4}{17} & 0 & \frac{9}{34} & 0 & 0 & 0 & 0 & 0 \\
0 & 0 & 0 & 0 & 0 & 0 & 0 & 0 \\
0 & 0 & -\frac{51}{86} & 0 & 0 & 0 & 0 & 0 \\
0 & 0 & \frac{1}{49} & 0 & 0 & 0 & 0 & 0
\end{array}\right] .
\end{aligned}
$$

Also in this case, $C(0)$ is singular. Here, it has rank 7 . We compute the SVD for $C(0)$, for which we get $\left(U^{*} E V\right)_{88}=-0.1384(1+i)$, and note that for this case the right hand side is in the column space of $C(0)$. Hence, all conditions in lemma 3.4 are satisfied.

The error estimates (3.5), (3.6) are derived as in the second order case. By the bound (3.20) in lemma 3.4 we conclude that fourth order accuracy is obtained.

\section{Numerical experiments}

In this section numerical experiments are performed to corroborate the theoretical results for the second and fourth order methods, and to expand the results to higher orders of accuracy. We consider if, and how, the accuracy of the solution is affected by the lower order approximation near the interface. Dispersion effects emerging when the solution is interacting with the interface over long times are also studied. In a last set of experiments we investigate the efficiency of using adaptive meshes.

Dirichlet boundary conditions are imposed weakly, i.e. using the SAT penalty technique, on exterior boundaries. For the exact form of the 
penalty parameters associated with the exterior boundaries we refer to [8]. Interface conditions are imposed weakly according to $(2.4),(2.5)$ in one spatial dimension and $(2.9),(2.10)$ in two dimensions, with the penalty parameters (2.7).

As in [8] we use a temporal discretization based on exponential integrators, where a smaller basis of the spatial discretization matrix is computed using a Krylov subspace method, see [10] for a thorough description. The size of the Krylov space is chosen adaptively in order to satisfy a given accuracy requirement, whereas the time step is fixed.

This type of method has been shown to be very efficient, especially for problems with high frequency oscillations in time [10]. The convergence rate of the temporal error is exponential and the Krylov method converges very quickly in each time step. The good convergence properties of the temporal discretization are important when investigating convergence rates of high order spatial discretizations. To measure high spatial accuracy very small time steps were needed, but tests with a time-discretization method with polynomial convergence rate (Runge-Kutta 4) led to prohibitively small time steps.

Discretizing using SBP operators with SAT penalty terms leads to a non-Hermitian spatial discretization matrix, such that for straightforward time-integration the Arnoldi method should be used. However, through a simple coordinate transformation, from $[u, v]^{T}$ to $[w, q]^{T}$,

$$
\left[\begin{array}{c}
w \\
q
\end{array}\right]=\left[\begin{array}{c}
P_{L}^{1 / 2} u \\
P_{R}^{1 / 2} v
\end{array}\right],
$$

the corresponding spatial discretization matrix for $[w, q]^{T}$ is Hermitian, and we can use the Lanczos method instead. We have used either the Arnoldi method or Lanczos iteration for all numerical simulations presented below.

The $l_{2}$ error and the max error are in each set of experiments computed as the norm of the difference between the numerical solution computed with gridsize $h, u^{h}$, and an exact solution $u_{e x}$, according to

$$
\begin{array}{r}
\left\|u^{h}-u_{e x}\right\|_{l_{2}}=\sqrt{h \sum_{j=1}^{N}\left|u_{j}^{h}-u_{e x, j}\right|^{2}}, \\
\left\|u^{h}-u_{e x}\right\|_{\infty}=\max _{1 \leq j \leq N}\left|u_{j}^{h}-u_{e x, j}\right|
\end{array}
$$


in one dimension and

$$
\begin{array}{r}
\left\|u^{h_{x}, h_{y}}-u_{e x}\right\|_{l_{2}}=\sqrt{h_{x} h_{y} \sum_{i=1}^{N_{1}} \sum_{j=1}^{N_{2}}\left|u_{i j}^{h_{x}, h_{y}}-u_{e x, i j}\right|^{2}}, \\
\left\|u^{h_{x}, h_{y}}-u_{e x}\right\|_{\infty}=\max _{1 \leq i \leq N_{1}} \max _{1 \leq j \leq N_{2}}\left|u_{i j}^{h_{x}, h_{y}}-u_{e x, i j}\right|,
\end{array}
$$

in two dimensions. Convergence rates are computed as

$$
q=\log \left(\frac{\left\|u^{h_{1}}-u_{e x}\right\|}{\left\|u^{h_{2}}-u_{e x}\right\|}\right) / \log \left(\frac{h_{1}}{h_{2}}\right) .
$$

Since there are two domains with different grid sizes, the errors are computed in each domain separately and added. In all two-dimensional experiments presented here, we set $h_{x}=h_{y}$.

\subsection{Convergence studies}

In these experiments a pulse is initiated in the coarse region and moves through the interface into the finer region. Figures 3 and 4 show a timeseries of the numerical solutions obtained with 4th and 8th order methods, respectively. In figure 5 the time-series are depicted in a semi-logarithmic scale. Note the the dominating error is situated at the interface for the 8th order method, whereas the dominating error for the 4th order method is following the pulse.

We carry out quantitative simulations on the domains $\Omega=[-12,12]$ and $\Omega=[-8,8] \times[-8,8]$, with an interface situated at $x=0$. The size of the computational domain is chosen such that the impact of the outer boundaries can be neglected for error comparisons. The simulations are run until time $T=0.25$ with the time step $\Delta t=2 \cdot 10^{-4}$ for orders 2 and 4 and $\Delta t=1 \cdot 10^{-4}$ or $\Delta t=5 \cdot 10^{-5}$ for order 6 and 8 , in order to keep the Krylov subspace in the time integration of reasonable size. The grid size ratio is $2: 1$ for simulations in both one and two dimensions. The initial data are chosen so that there exists an exact solution to the problem without boundaries that the numerical solution can be compared against. In tables $1-8$, results are listed. Here, $N$ is normalized to correspond to the same grid size for simulations in both one and two dimensions.

According to the theoretical predictions, spatial discretization orders 2 and 4 should lead to overall accuracies of the same orders. This behavior is verified in tables $1-2$, and tables 5-6, for one- and two-dimensional simulations, respectively. Results for orders 6 and 8 are displayed in tables 3-4 and $7-8$, respectively. We note that the maximum errors converge with 5 th and 6 th order, respectively. These convergence results are of order $m+2$, 
which is the best we could hope for. Remember that the right hand side in (3.17) will be $\mathcal{O}\left(h^{m+2}\right)$ due to the second order derivative in the TDSE. If $C(0)$ is nonsingular, or if lemma 3.4 can be used as in the fourth order case, an accuracy of order $m+2$ will be obtained.

However, the $l_{2}$ error for the 6 th order discretization converges with a higher rate. The reason for this is that in the particular experiments for which the results are displayed in table 3 , the interface error does not dominate until the last grid refinement level, where we get 5th order accuracy in the max norm. We have conducted experiments where the solution is more localized to the interface at the final time, so that the interface error is more prominent and has a larger impact on the $l_{2}$ error. In those cases we have seen a decrease in the convergence rate also in the $l_{2}$ norm.

\begin{tabular}{ccccc}
\hline$N$ & $\left\|u-u_{e x}\right\|_{l_{2}}$ & $\left\|u-u_{e x}\right\|_{\infty}$ & $q_{l_{2}}$ & $q_{\infty}$ \\
\hline 101 & 1.15 & $8.15 \cdot 10^{-1}$ & - & - \\
201 & $3.01 \cdot 10^{-1}$ & $2.16 \cdot 10^{-1}$ & 1.96 & 1.94 \\
401 & $7.52 \cdot 10^{-2}$ & $5.40 \cdot 10^{-2}$ & 2.01 & 2.01 \\
801 & $1.88 \cdot 10^{-2}$ & $1.35 \cdot 10^{-2}$ & 2.01 & 2.01 \\
\hline
\end{tabular}

Table 1: Errors for pulse traveling through interface in 1D, 2nd order spatial discretization. $N$ denotes the normalized number of grid points in the in the coarse domain.

\begin{tabular}{ccccc}
\hline$N$ & $\left\|u-u_{e x}\right\|_{l_{2}}$ & $\left\|u-u_{e x}\right\|_{\infty}$ & $q_{l_{2}}$ & $q_{\infty}$ \\
\hline 101 & $1.24 \cdot 10^{-1}$ & $7.35 \cdot 10^{-2}$ & - & - \\
201 & $7.46 \cdot 10^{-3}$ & $4.72 \cdot 10^{-3}$ & 4.11 & 4.02 \\
401 & $4.40 \cdot 10^{-4}$ & $2.96 \cdot 10^{-4}$ & 4.11 & 4.03 \\
801 & $2.67 \cdot 10^{-5}$ & $1.84 \cdot 10^{-5}$ & 4.06 & 4.02 \\
\hline
\end{tabular}

Table 2: Errors for pulse traveling through interface in 1D, 4th order spatial discretization. $N$ denotes the normalized number of grid points in the in the coarse domain.

\begin{tabular}{ccccc}
\hline$N$ & $\left\|u-u_{e x}\right\|_{l_{2}}$ & $\left\|u-u_{e x}\right\|_{\infty}$ & $q_{l_{2}}$ & $q_{\infty}$ \\
\hline 201 & $6.94 \cdot 10^{-4}$ & $3.37 \cdot 10^{-4}$ & - & - \\
401 & $7.55 \cdot 10^{-6}$ & $3.04 \cdot 10^{-6}$ & 6.52 & 6.79 \\
801 & $8.41 \cdot 10^{-8}$ & $3.80 \cdot 10^{-8}$ & 6.49 & 6.32 \\
1601 & $1.04 \cdot 10^{-9}$ & $6.54 \cdot 10^{-10}$ & 6.34 & 5.86 \\
3201 & $1.08 \cdot 10^{-11}$ & $2.04 \cdot 10^{-11}$ & 6.58 & 5.00 \\
\hline
\end{tabular}

Table 3: Errors for pulse traveling through interface in 1D, 6th order spatial discretization. 


\begin{tabular}{ccccc}
\hline$N$ & $\left\|u-u_{e x}\right\|_{l_{2}}$ & $\left\|u-u_{e x}\right\|_{\infty}$ & $q_{l_{2}}$ & $q_{\infty}$ \\
\hline 201 & $2.75 \cdot 10^{-2}$ & $9.93 \cdot 10^{-2}$ & - & - \\
401 & $1.61 \cdot 10^{-6}$ & $7.53 \cdot 10^{-6}$ & 14.06 & 13.69 \\
801 & $1.57 \cdot 10^{-8}$ & $1.14 \cdot 10^{-7}$ & 6.68 & 6.05 \\
1601 & $1.77 \cdot 10^{-10}$ & $1.81 \cdot 10^{-9}$ & 6.47 & 5.97 \\
\hline
\end{tabular}

Table 4: Errors for pulse traveling through interface in 1D, 8th order spatial discretization.

\begin{tabular}{ccccc}
\hline$N_{1}$ & $\left\|u-u_{e x}\right\|_{l_{2}}$ & $\left\|u-u_{e x}\right\|_{\infty}$ & $q_{l_{2}}$ & $q_{\infty}$ \\
\hline 51 & 2.80 & 1.75 & - & - \\
101 & 1.28 & $7.79 \cdot 10^{-1}$ & 1.14 & 1.18 \\
151 & $5.95 \cdot 10^{-1}$ & $3.55 \cdot 10^{-1}$ & 1.91 & 1.96 \\
201 & $3.37 \cdot 10^{-1}$ & $1.99 \cdot 10^{-1}$ & 1.99 & 2.01 \\
\hline
\end{tabular}

Table 5: Errors for pulse traveling through interface in 2D, 2nd order spatial discretization. $N_{1}$ denotes the number of grid points in the $x$-direction in the coarse domain.

\begin{tabular}{ccccc}
\hline$N_{1}$ & $\left\|u-u_{e x}\right\|_{l_{2}}$ & $\left\|u-u_{e x}\right\|_{\infty}$ & $q_{l_{2}}$ & $q_{\infty}$ \\
\hline 51 & 1.54 & $8.78 \cdot 10^{-1}$ & - & - \\
101 & $1.39 \cdot 10^{-1}$ & $7.85 \cdot 10^{-2}$ & 3.53 & 3.53 \\
151 & $2.71 \cdot 10^{-2}$ & $1.52 \cdot 10^{-2}$ & 4.06 & 4.08 \\
201 & $8.35 \cdot 10^{-3}$ & $4.69 \cdot 10^{-3}$ & 4.11 & 4.11 \\
301 & $1.59 \cdot 10^{-3}$ & $8.94 \cdot 10^{-4}$ & 4.10 & 4.11 \\
\hline
\end{tabular}

Table 6: Errors for pulse traveling through interface in 2D, 4th order spatial discretization. $N_{1}$ denotes the number of grid points in the $x$-direction in the coarse domain.

\begin{tabular}{ccccc}
\hline$N_{1}$ & $\left\|u-u_{e x}\right\|_{l_{2}}$ & $\left\|u-u_{e x}\right\|_{\infty}$ & $q_{l_{2}}$ & $q_{\infty}$ \\
\hline 201 & $7.80 \cdot 10^{-4}$ & $4.52 \cdot 10^{-4}$ & - & - \\
301 & $7.99 \cdot 10^{-5}$ & $7.79 \cdot 10^{-5}$ & 5.64 & 4.35 \\
401 & $8.45 \cdot 10^{-6}$ & $4.76 \cdot 10^{-6}$ & 7.83 & 9.75 \\
501 & $1.97 \cdot 10^{-6}$ & $1.11 \cdot 10^{-6}$ & 6.54 & 6.54 \\
601 & $6.03 \cdot 10^{-7}$ & $3.39 \cdot 10^{-7}$ & 6.51 & 6.51 \\
\hline
\end{tabular}

Table 7: Errors for pulse traveling through interface in 2D, 6th order spatial discretization. $N_{1}$ denotes the number of grid points in the $x$-direction in the coarse domain. 


\begin{tabular}{ccccc}
\hline$N_{1}$ & $\left\|u-u_{e x}\right\|_{l_{2}}$ & $\left\|u-u_{e x}\right\|_{\infty}$ & $q_{l_{2}}$ & $q_{\infty}$ \\
\hline 201 & $3.08 \cdot 10^{-2}$ & $1.25 \cdot 10^{-1}$ & - & - \\
301 & $2.77 \cdot 10^{-5}$ & $1.28 \cdot 10^{-4}$ & 17.37 & 17.04 \\
401 & $1.80 \cdot 10^{-6}$ & $9.44 \cdot 10^{-6}$ & 9.53 & 9.10 \\
501 & $3.98 \cdot 10^{-7}$ & $2.46 \cdot 10^{-6}$ & 6.79 & 6.03 \\
601 & $1.16 \cdot 10^{-7}$ & $7.99 \cdot 10^{-7}$ & 6.76 & 6.19 \\
\hline
\end{tabular}

Table 8: Errors for pulse traveling through interface in 2D, 8th order spatial discretization. $N_{1}$ denotes the number of grid points in the $x$-direction in the coarse domain.
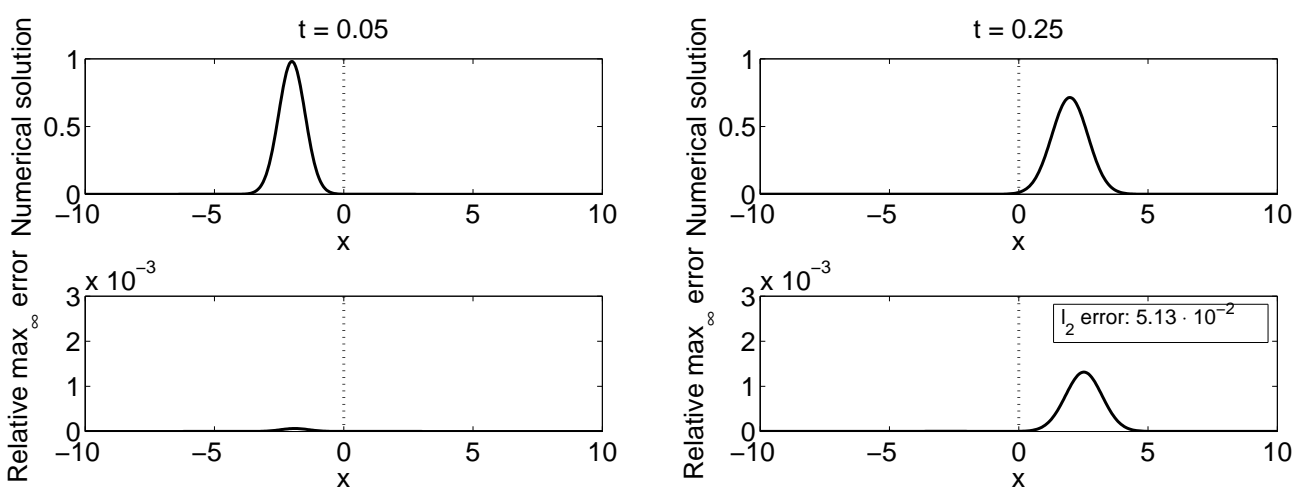

Figure 3: Numerical solution traveling through interface for 4th order spatial discretizations. Relative max error with respect to exact solution is depicted underneath. 

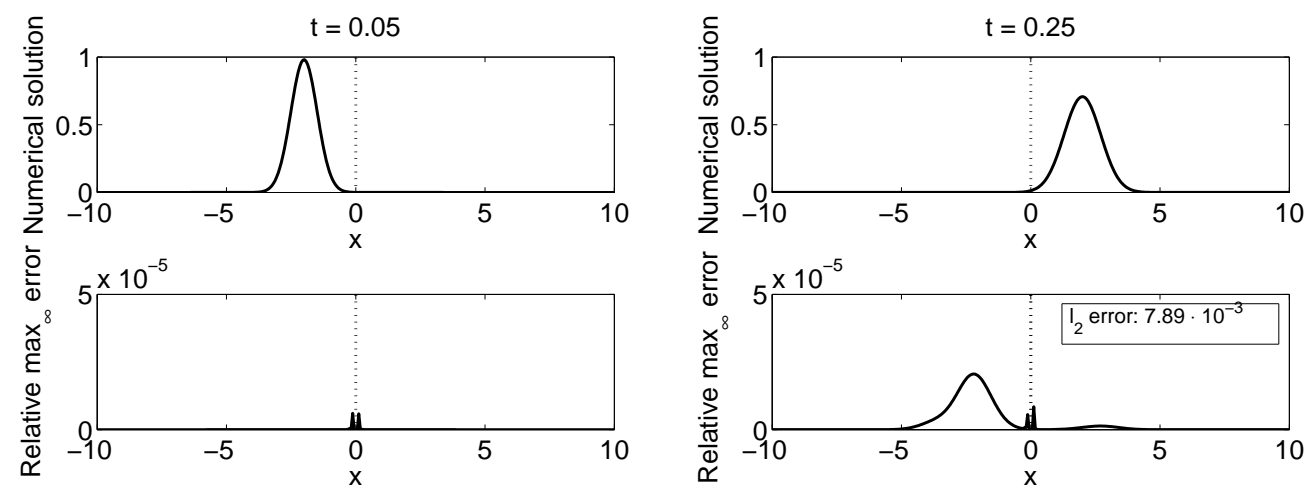

Figure 4: Numerical solution traveling through interface for 8th order spatial discretizations. Relative max error with respect to exact solution is depicted underneath.
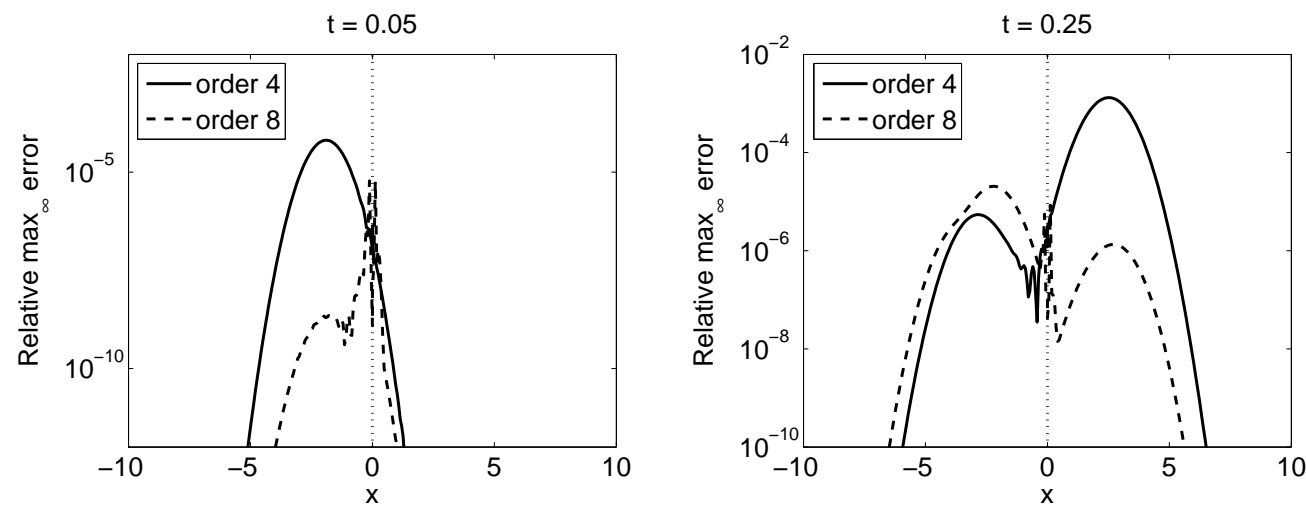

Figure 5: Relative max error with respect to exact solution for 4th and 8th order spatial discretizations, respectively. Semi-logarithmic scale.

\subsection{Long time effects}

Long time effects of a pulse that travels across the interface repeatedly are examined with the one-dimensional model problem

$$
U_{t}=i U_{x x}+V U,
$$

where the potential $V$ is an harmonic oscillator potential $V=\frac{\omega^{2}}{4} x^{2}$. An exact solution to this problem exists. It consists of a pulse that oscillates in 
space. We compute the error at times $T=\frac{2 \pi n}{\omega}$, with $n=1,2, \ldots$, for which the exact solution is equal to the initial condition if this initial condition is chosen as

$$
U(x, 0)=e^{-\frac{\omega}{4}\left(x-x_{0}\right)^{2}}, x_{0}=-\frac{2}{\omega} .
$$

Figures 6 and 7 display the $l_{2}$ error as a function of time for $n=1, \ldots, 10$, $\omega=4 \pi$, for experiments with and without interface. The experiments are run until $T=10$, using a time step of size $\Delta t=1 \cdot 10^{-3}$, with $N=301$.

Figure 6 depicts the errors in a semi-logarithmic scale, with one figure for each spatial order. In figure 7 the errors with respect to time for different orders are depicted on the same scale, the left figure for simulations with an interface and the right figure without interface. We see from the figures that the impact of the interface increases with the order. This is not so surprising, since we have shown that for orders two and four the accuracy with the interface is of the same order as without interface. For orders six and eight, however, we expect to get lower accuracy due to the lower order approximation close to the interface.

From figure 7 we see that going to 6 th order method from 4 th order does pay off in terms of accuracy. However, we also need to consider the computational cost in order to investigate if it is more efficient to use high order methods. We investigate this below. 

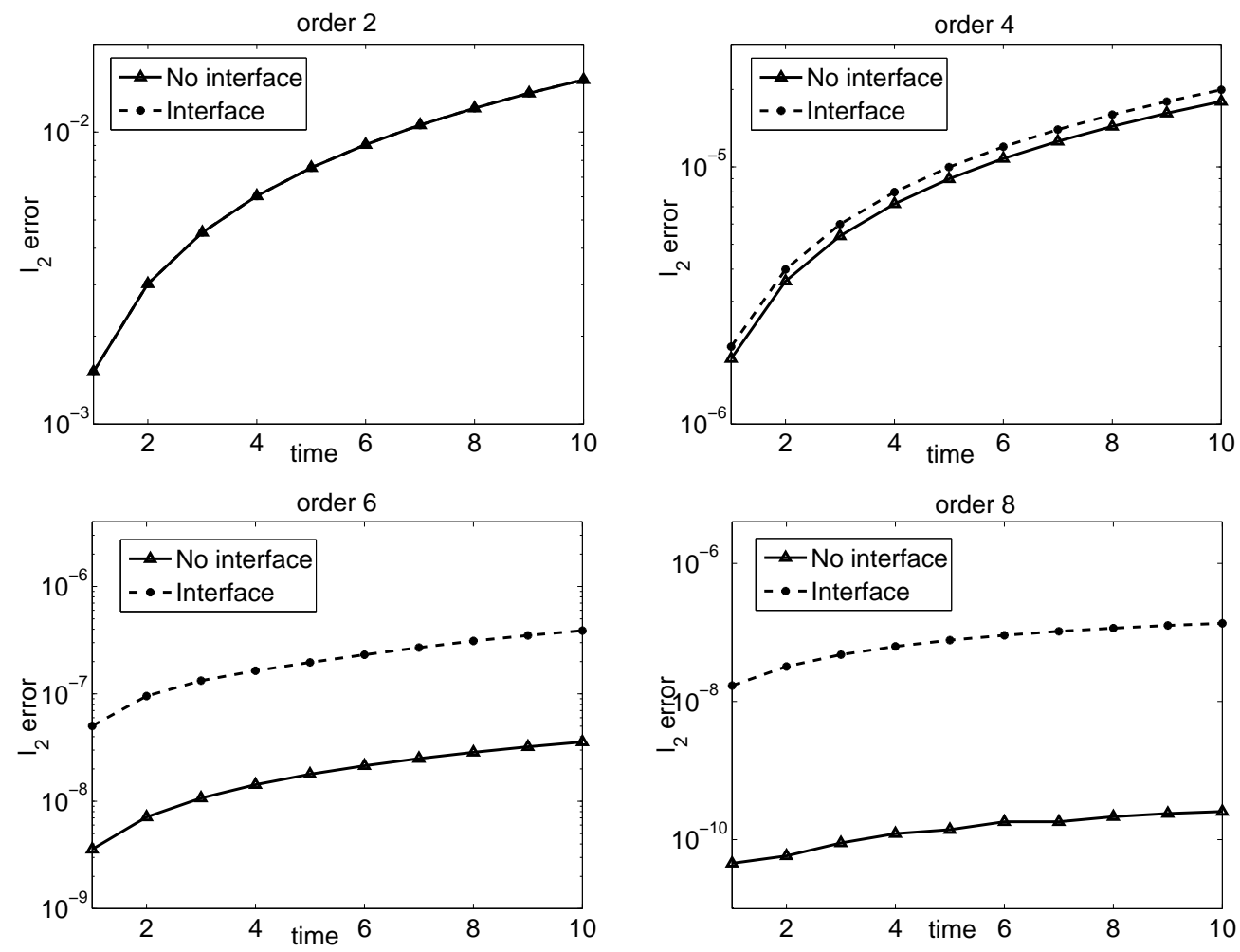

Figure 6: Long time behavior of $l_{2}$ error as a function of time, with and without interface. Spatial discretizations of order 2, 4,6 and 8, respectively. 

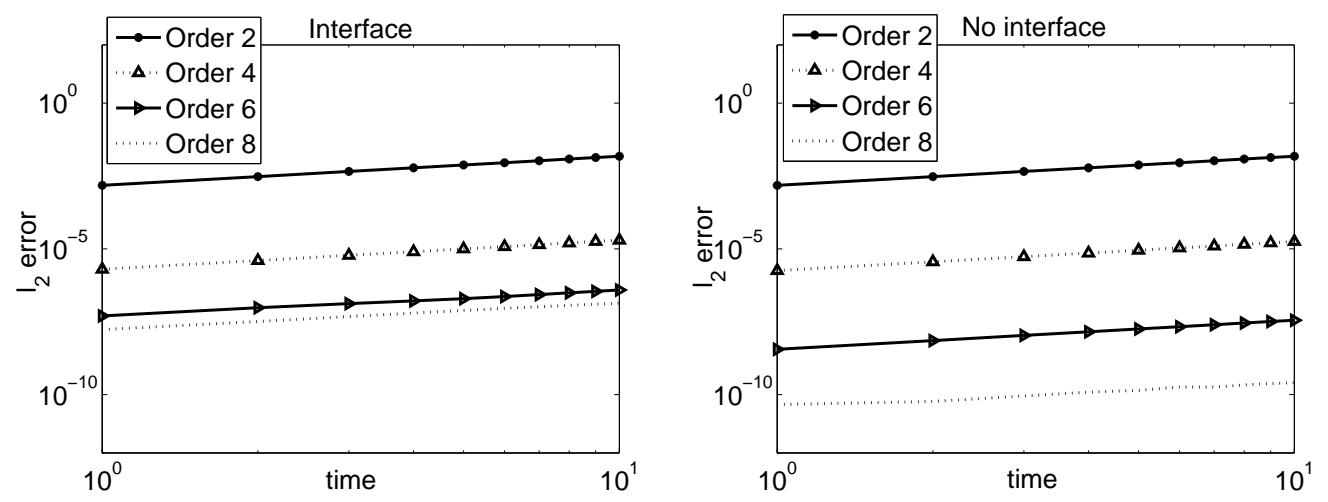

Figure 7: Long time behavior of $l_{2}$-error as a function of time, logarithmic scale. Left figure shows errors for experiments with interface, right figure without interface. Spatial discretizations of order 2, 4, 6 and 8, respectively.

\subsection{Efficiency of adaptivity}

We illustrate the potential efficiency gain when using high order finite difference methods on adaptive meshes with numerical experiments on the model problem

$$
U_{t}=i U_{x x}+i U_{y y}+V U, \quad V=\frac{\omega^{2}}{4}\left(x^{2}+y^{2}\right),
$$

with initial condition

$$
e^{i k_{x} x-\frac{\omega}{4} x^{2}-\frac{\omega}{4} y^{2}}
$$

where $\omega=10$ and $k_{x}=-5$. In the exact solution the initial pulse, centered at the origin, oscillates in the $x$-direction. The experiments are conducted on the domain $[-7.5,7.5] \times[-7.5,7.5]$ and run until $\mathrm{T}=0.05$.

The structure of the adaptive mesh is depicted in figure 8. It contains nine grid patches where the middle one is refined one more time in each direction compared to the surrounding patches. A closer view of the mesh in the lower left corner is given in figure 8, right figure. Figure 9 shows a contour graph of the initial solution. Here, we have chosen the adaptive mesh such that the bulk of the solution is confined in the fine grid block. All grid blocks are coupled with interfaces as described earlier in this paper.

We set the global tolerance to $10^{-3}$, and for each order find the largest $h$ that meets the tolerance. Next, we count how many floating point operations that is required when applying the spatial discretization matrix to the numerical solution as a measure of the work required in each time step. 
This is done both for an adaptive mesh and for a uniform mesh without interfaces. The actual $l_{2}$ error compared to the exact solution, the required number of grid points, and the number of operations in one matrix-vector multiplication is given in table 9 .

Looking at the results for the uniform meshes it is clear that employing higher order methods pays off. There is an additional gain when adaptivity is used, which decreases with order. However, we do not see an efficiency gain if we use an adaptive 8th order method rather than the adaptive 6 th order method. Experiments not reported in this paper indicate that a stricter tolerance leads to increased efficiency of the 8th order adaptive discretization. In higher dimensions we expect that an adaptive grid would increase efficiency even more.

Here, we have only considered the impact of the spatial discretization. In the experiments we have seen that the presence of the interfaces leads to slower convergence of the Krylov subspace method in each time step. In order to fully understand the efficiency of the overall discretization further investigations are required.
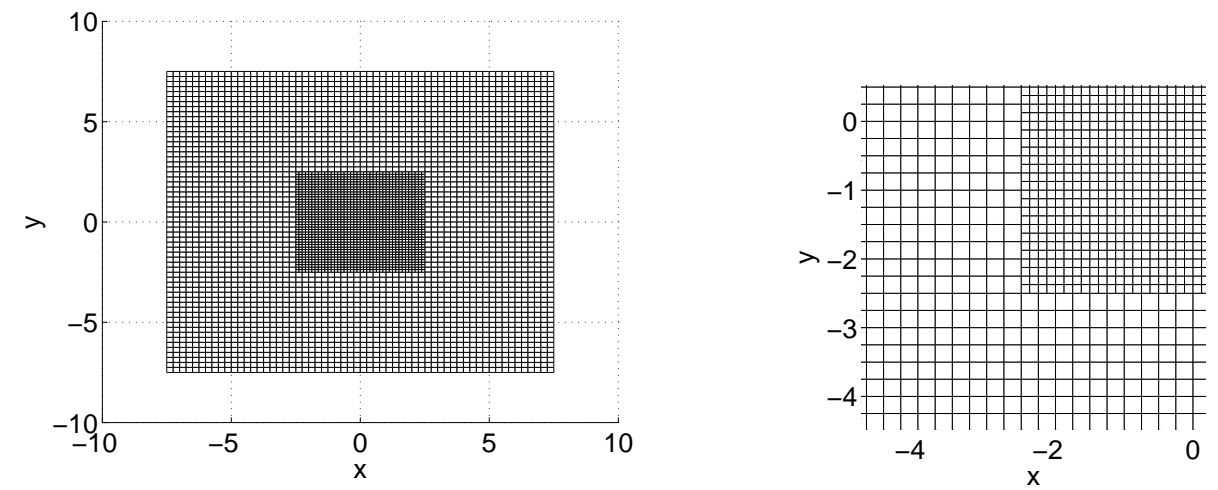

Figure 8: The left figure depicts the computational domain discretized with an adaptive grid composed of nine grid patches. The coarser blocks each contain $21 \times 21$ grid points and the finer block $41 \times 41$ grid points. A closer caption of the lower left corner of the nonconforming interface is given in the right figure. 


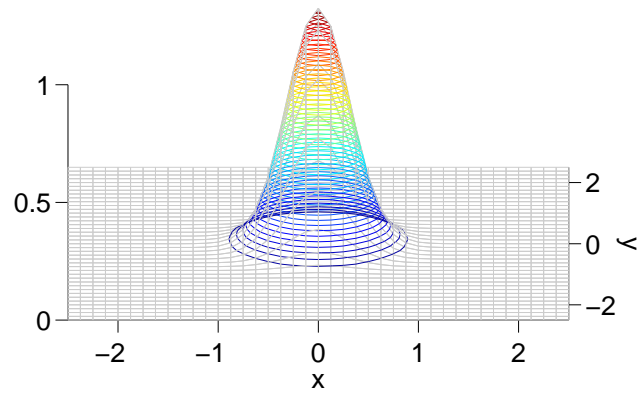

Figure 9: Contour plot of the initial function on the fine part of the adaptive mesh.

\begin{tabular}{ccccc}
\hline order & mesh & $\left\|u-u_{e x}\right\|_{l_{2}}$ & no. grid points & no. floating point op. \\
\hline 2 & uniform & $9.96 \cdot 10^{-4}$ & 2647129 & 23837177 \\
2 & adaptive & $9.97 \cdot 10^{-4}$ & 886721 & 8154241 \\
4 & uniform & $9.37 \cdot 10^{-4}$ & 64009 & 1082081 \\
4 & adaptive & $9.37 \cdot 10^{-4}$ & 22017 & 418313 \\
6 & uniform & $8.53 \cdot 10^{-4}$ & 21025 & 525625 \\
6 & adaptive & $8.61 \cdot 10^{-4}$ & 7401 & 242481 \\
8 & uniform & $6.39 \cdot 10^{-4}$ & 13225 & 436425 \\
8 & adaptive & $8.90 \cdot 10^{-4}$ & 4721 & 244817 \\
\hline
\end{tabular}

Table 9: Number of floating point operation in one matrix vector operation for operators for a uniform mesh and an adaptive mesh. Tolerance is $10^{-3}$.

\section{Conclusions}

In this paper we consider stability and accuracy at grid interfaces for the Schrödinger equation using the SBP-SAT methodology. We construct penalty parameters that lead to stable nonconforming grid interfaces in arbitrary many dimensions.

We consider accuracy of the grid interface coupling in one dimension for SBP operators of formal order two and four, and show that the global order of accuracy is of order two and four, respectively. Higher orders of accuracy, with interior order $2 m$, are investigated through numerical simulations that show that the global order of accuracy is $m+2$. Thus, 5 th and 6 th order of accuracy, respectively, is obtained for orders six and eighth, respectively. 
Numerical experiments conducted with an adaptive multiblock configuration grid in two dimensions are compared to simulations on a uniform grid. The experiments show that higher order methods are more efficient. For a given tolerance $\left(10^{-3}\right)$ the adaptivity leads to an additional gain in efficiency up to 6 th order. For higher dimensions we expect the gain to be more prominent.

In order to determine the optimal order of accuracy in terms of efficiency it would be interesting to consider the time-stepping as well as parallel implementation issues, such as communication between processors and memory requirements, in a multi-block configuration. An adaptive solver based on the interface treatment and accuracy analysis in this paper will be implemented.

\section{Acknowledgements}

The authors would like to thank Katharina Kormann for help with the time-stepping method. This work has been partially financed by Anna Maria Lundins stipendiefond and the Swedish research council. The computations were performed on resources provided by SNIC through Uppsala Multidisciplinary Center for Advanced Computational Science (UPPMAX) under Project p2005005. 


\section{References}

[1] M. J. Berger, J. Oliger, Adaptive mesh refinement for hyperbolic partial differential equations, J. Comput. Phys. 53 (1984), 484-512.

[2] Q. Stout, D. De Zeeuw, T. Gombosi, C. Groth, H. Marshall, K. Powell, Adaptive blocks: A high-performance data structure, in Proceedings, Supercomputing '97 (1997).

[3] J. Rantakokko, M. Thuné, (2009). Parallel structured adaptive mesh refinement. In Troubec, R. et al (eds.), Parallel Computing, Springer Verlag, London, pp. 147-173.

[4] D. Kosloff, R. Kosloff. (1983). A Fourier method solution for the time dependent Schrödinger equation as a tool in molecular dynamics, J. Comput. Phys. 52, 35-53.

[5] M. H. Carpenter, D. Gottlieb, S. Abarbanel. (1994). Time-stabe boundary conditions for finite difference schemes solving hyperbolic systems: Methodology and application to high-order compact schemes. J. Comput. Phys. 111, 220-236.

[6] M. H. Carpenter, J. Nordström, D. Gottlieb, A stable and conservative interface treatment of arbitrary spatial accuracy, J. Comput. Phys. 148 (1999), 341-365.

[7] K. Mattsson, M. H. Carpenter, Stable and accurate interpolation operators for high-order multiblock finite difference methods, SIAM J. Sci. Comput. 32 (2010), 4, 2298-2320.

[8] A. Nissen, G. Kreiss, M. Gerritsen, High order stable finite difference methods for the Schrödinger equation, Technical report / Department of Information Technology, Uppsala University nr 2011-014, 2011.

[9] K. Mattsson, J. Nordström, Summation by parts operators for finite difference approximations of second derivatives, J. Comput. Phys. 199 (2004), 503-540.

[10] K. Kormann, S. Holmgren and H. O. Karlsson, Accurate time propagation for the Schrödinger equation with an explicitly time-dependent Hamiltonian, J. Chem Phys., 128 (2008), 184101-184101-11.

[11] B. Gustafsson, H-O. Kreiss and J. Oliger, Time-dependent problems and difference methods, John Wiley \& Sons, Inc., 1995. 